Supporting Information

\title{
Palladium-Catalyzed, Enantioselective Desymmetrization of $N$-Acylaziridines with Indoles
}

\author{
Kinney Van Hecke, Tyler R. Benton, Michael Casper, Dustin Mauldin, Brandon Drake, and \\ Jeremy B. Morgan* \\ University of North Carolina Wilmington, Department of Chemistry and Biochemistry, Dobo Hall, \\ Wilmington, NC 28403, USA \\ Corresponding author's email: morganj@uncw.edu
}

Crystallographic Data for $[(R)$-DM-SEGPHOS $] \mathrm{Pd}\left(\mathrm{BF}_{4}\right)_{2} \bullet 2 \mathrm{H}_{2} \mathrm{O}(\boldsymbol{9}, \mathrm{KWV}-\mathrm{II}-79) \ldots \ldots \ldots \ldots . . \mathrm{S} 2-\mathrm{S} 11$

Crystallographic Data for Tryptamine 13 (KWV-II-172)..............................S12-S20 
Submitted by: Jeremy Morgan

Solved by: Thomas Pickel, John Bacsa

Sample ID: KWV-II-79

\section{Crystal Data and Experimental}

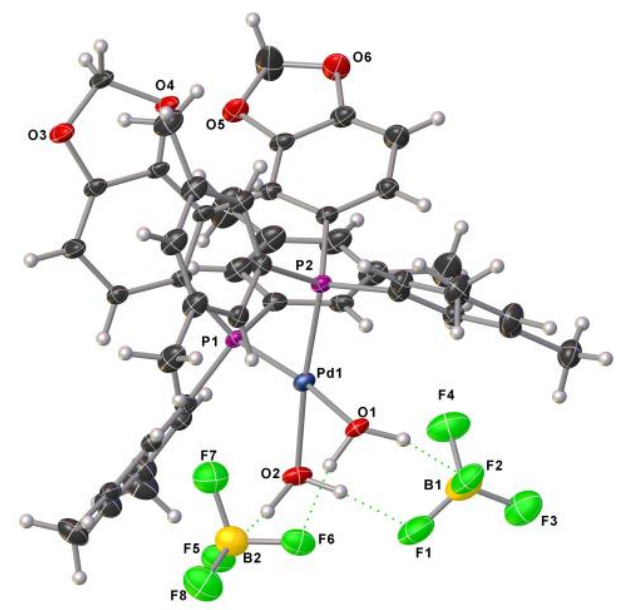

Experimental. Single yellow plate-shaped crystals of (KWV-II-79) were recrystallised from DCM and MtBEby vapor diffusion. A suitable crystal $(0.53 \times 0.23 \times 0.11 \mathrm{~mm})$ was selected and mounted on a loop with paratone oil on a Bruker APEX-II CCD diffractometer. The crystal was cooled to $T=100(2) \mathrm{K}$ during data collection. The structure was solved with the ShelXT-2014/4 (Sheldrick, 2015) structure solution program using combined Patterson and dual-space recycling methods and by using Olex2 (Dolomanov et al., 2009) as the graphical interface. The crystal structure was refined with version 2014/7 of ShelXL (Sheldrick, 2014) using Least Squares minimisation.

Crystal Data. $\mathrm{C}_{46} \mathrm{H}_{48} \mathrm{~B}_{2} \mathrm{~F}_{8} \mathrm{O}_{6} \mathrm{P}_{2} \mathrm{Pd}, \quad M_{r}=1038.80$, orthorhombic, $\mathrm{C} 222_{1}$ (No. 20), $\mathrm{a}=12.986(3) \AA, \mathrm{b}=$ 23.963(7) $\AA, \quad \mathrm{c}=32.862(7) \AA, \quad \alpha=\beta=\gamma=90^{\circ}, \quad V=$ 10226(4) $\AA^{3}, T=100(2) \mathrm{K}, Z=8, Z^{\prime}=1, \mu\left(\mathrm{MoK}_{\alpha}\right)=0.497$ $\mathrm{mm}^{-1}, 42389$ reflections measured, 9632 unique $\left(R_{\text {int }} \#=\right.$ $0.0717)$ which were used in all calculations. The final $w R_{2}$

\section{Compound}

Formula

$D_{\text {calc. }} / \mathrm{g} \mathrm{cm}^{-3}$

$\mu / \mathrm{mm}^{-1}$

Formula Weight

Colour

Shape

Max Size/mm

Mid Size $/ \mathrm{mm}$

Min Size/mm

$T / \mathrm{K}$

Crystal System

Flack Parameter

Hooft Parameter

Space Group

a/Å

$b / \AA$

$c / \AA$

$\alpha /^{\circ}$

$\beta /^{\circ}$

$\gamma /{ }^{\circ}$

$\mathrm{V} / \AA^{3}$

$Z$

$Z^{\prime}$

$\Theta_{\min } /^{\circ}$

$\Theta_{\max } /^{\circ}$

Measured Refl.

Independent Refl.

Reflections I $>2 \sigma(\mathrm{I})$

$R_{\text {int }}$

Parameters

Restraints

Largest Peak

Deepest Hole

GooF

$w R_{2}$ (all data)

$w R_{2}$

$R_{1}$ (all data)

$R_{1}$
KWV-II-79

$\mathrm{C}_{46} \mathrm{H}_{48} \mathrm{~B}_{2} \mathrm{~F}_{8} \mathrm{O}_{6} \mathrm{P}_{2} \mathrm{Pd}$

1.349

0.497

1038.80

yellow

plate

0.53

0.23

0.11

100(2)

orthorhombic

$0.024(14)$

$0.019(14)$

C222 1

12.986(3)

23.963(7)

32.862(7)

90

90

90

10226(4)

8

1

1.239

25.665

42389

9632

8315

0.0717

606

595

1.948

$-1.855$

1.176

0.1723

0.1675

0.0892

0.0773

was 0.1723 (all data) and $R_{1}$ was $0.0773(\mathrm{I}>2 \sigma(\mathrm{I}))$. 
Structure Quality Indicators

Reflections:

$\mathrm{d} \min (\mathrm{Mo})$

$0.82^{1 / \pi}$

$\left.13.4\right|^{\text {Rint }}$

$7.17 \%$ complete

$99 \%$

Refinement:

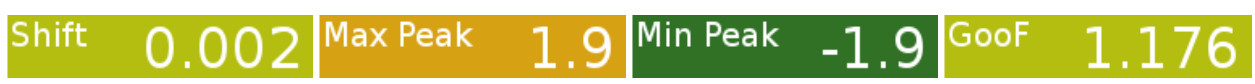

Experimental Extended. A yellow plate-shaped crystal with dimensions $0.53 \times 0.23 \times 0.11 \mathrm{~mm}$ was mounted on a loop with paratone oil. X-ray diffraction data were collected using a Bruker APEX-II CCD diffractometer equipped with an Oxford Cryosystems low-temperature apparatus operating at $T=100(2) \mathrm{K}$.

Data were measured using $\phi$ and $\omega$ scans of $0.50^{\circ}$ per frame for $30.00 \mathrm{~s}$ using $\mathrm{MoK}_{\alpha}$ radiation (fine-focus sealed tube, $45 \mathrm{kV}, 35 \mathrm{~mA}$ ). The total number of runs and images was based on the strategy calculation from the program APEX2 (Bruker). The maximum resolution achieved was $\Theta=25.67^{\circ}$.

Unit cell indexing was performed by using the APEX2 (Bruker) software and refined using SAINT (Bruker, V8.34A, 2013) on 9916 reflections, 23\% of the observed reflections. Data reduction, scaling and absorption corrections were performed using SAINT (Bruker, V8.34A, 2013) and SADABS-2014/5 (Bruker, 2014). $w R_{2}$ (int) was 0.1420 before and 0.0625 after correction. The ratio of minimum to maximum transmission is 0.7271.The $\lambda / 2$ correction factor is 0.00150 . The final completeness is $99.3 \%$ out to $25.67^{\circ}$ in $\Theta$. The absorption coefficient $\mu$ of this material is $0.497 \mathrm{~mm}^{-1}$ at this wavelength $(\lambda=0.71073 \AA)$ and the minimum and maximum transmissions are 0.6984 and 0.9605 .

The structure was solved in the space group P1 with the ShelXT-2014/4 (Sheldrick, 2015) structure solution program using combined Patterson and dual-space recycling methods. The space group C222 1 (\# 20) was determined by ShelXT-2014/4 (Sheldrick, 2015). The crystal structure was refined by Least Squares using version 2014/7 of ShelXL (Sheldrick, 2014). All non-hydrogen atoms were refined anisotropically. Hydrogen atom positions were calculated geometrically, water molecule hydrogen atom positions were derived by considering the hydrogen bond environment and refined using restraints or the riding model. The Flack parameter was refined to 0.024(14), confirming the absolute stereochemistry. Determination of absolute structure using Bayesian statistics on Bijvoet differences using the program within PLATON (Spek, 2003) also report that we have the correct enantiomer based on this comparison. Note: The Flack parameter is used to determine chirality of the crystal studied, the value should be near 0 , a value of 1 mans that the stereochemistry is wrong and the model should be inverted. A value of 0.5 means that the crystal consists of a racemic mixture of the two enantiomers. 


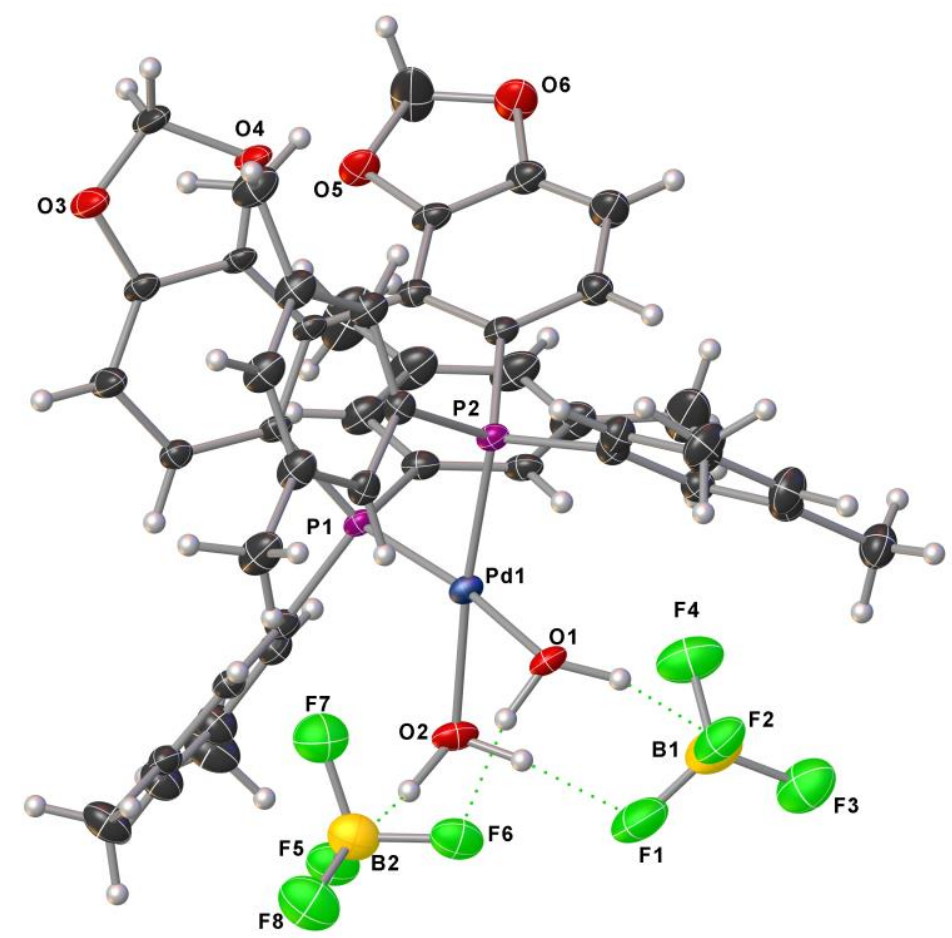

Figure 1:

Plot of the asymmetric unit with thermal ellipsoids at 50\% probability level.

\section{Data Plots: Diffraction Data}
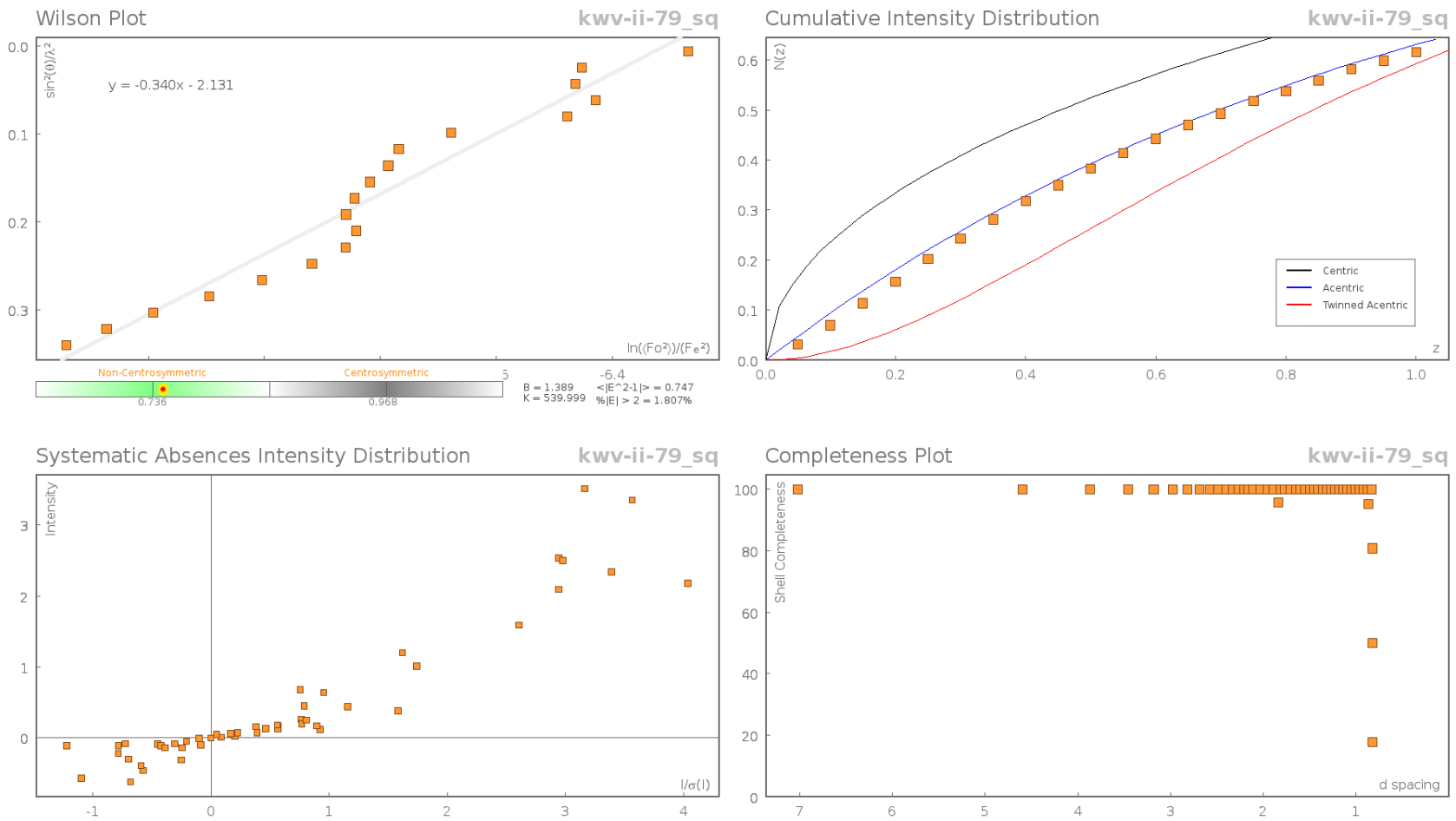


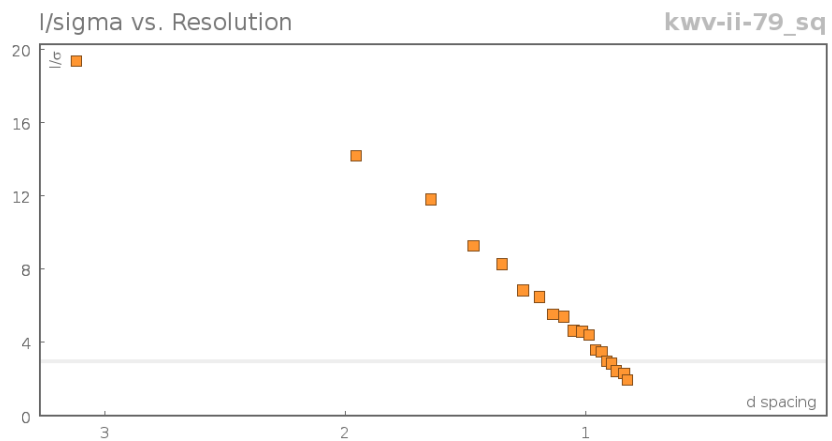

\section{Data Plots: Refinement and Data}
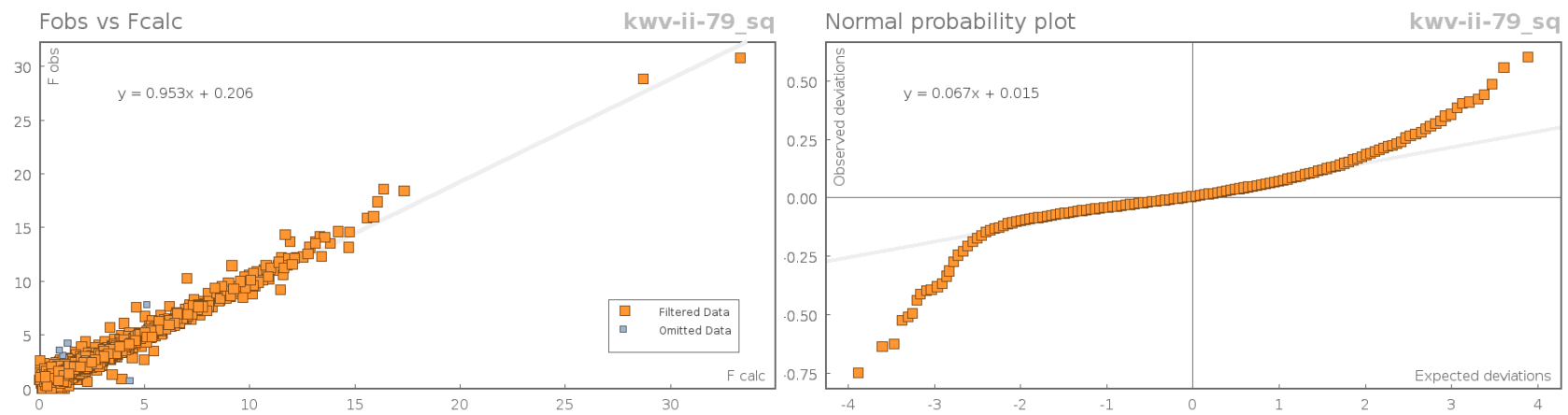

\section{Reflection Statistics}

Total reflections (after filtering)

Completeness

$\mathrm{hkl}_{\max }$ collected

hklmax $_{\text {max }}$

Lim dmax collected

$\mathrm{d}_{\text {max }}$ used

Friedel pairs

Inconsistent equivalents

$\mathrm{R}_{\text {sigma }}$

Omitted reflections

Multiplicity

Removed systematic absences 53
42486

0.992

$(15,29,39)$

$(15,29,39)$

100.0

16.43

11895

0

0.0678

0

(20206, 9444, 1084, 35)

53

$\begin{array}{ll}\text { Unique reflections } & 9632 \\ \text { Mean I/ } \sigma & 13.36 \\ \mathrm{hkl}_{\text {min }} \text { collected } & (-15,-28,-38) \\ \mathrm{hkl}_{\text {min }} \text { used } & (-15,0,0) \\ \text { Lim d } \mathrm{d}_{\text {min }} \text { collected } & 0.36 \\ \mathrm{~d}_{\text {min }} \text { used } & 0.82 \\ \text { Friedel pairs merged } & 0 \\ \text { Rint } & 0.0717 \\ \text { Intensity transformed } & 0 \\ \text { Omitted by user (OMIT hkl) } & 44 \\ \text { Maximum multiplicity } & 13 \\ \text { Filtered off (Shel/OMIT) } & 0\end{array}$

Table 1: Fractional Atomic Coordinates $\left(\times 10^{4}\right)$ and Equivalent Isotropic Displacement Parameters $\left(\AA^{2} \times 10^{3}\right)$ for KWV-II-79. Ueq is defined as $1 / 3$ of of the trace of the orthogonalised Uij.

\begin{tabular}{lllll}
\hline Atom & \multicolumn{1}{c}{$\mathbf{x}$} & \multicolumn{1}{c}{$\mathbf{y}$} & \multicolumn{1}{c}{$\mathbf{z}$} & Ueq \\
\hline Pd1 & $2469.3(7)$ & $6273.0(3)$ & $6252.8(2)$ & $18.8(2)$ \\
P2 & $3349(2)$ & $6744.6(11)$ & $5778.1(9)$ & $16.3(5)$ \\
P1 & $2748.5(19)$ & $6952.8(11)$ & $6700.5(9)$ & $15.7(5)$ \\
01 & $2393(6)$ & $5550(3)$ & $5868(2)$ & $20.8(15)$ \\
F6 & $2504(7)$ & $4658(3)$ & $6363(2)$ & $40.2(14)$ \\
O4 & $5714(6)$ & $8269(3)$ & $6175(2)$ & $24.8(16)$ \\
F2 & $453(6)$ & $5367(3)$ & $5629(3)$ & $44.8(17)$ \\
F5 & $2512(8)$ & $4932(3)$ & $7019(2)$ & $41.7(15)$ \\
05 & $3682(6)$ & $8820(3)$ & $6291(3)$ & $28.8(16)$
\end{tabular}




\begin{tabular}{|c|c|c|c|c|}
\hline Atom & $\mathrm{x}$ & $y$ & $\mathrm{z}$ & Ueq \\
\hline$\overline{\text { F1 }}$ & $-152(6)$ & $5425(3)$ & $6273(3)$ & $55.4(18)$ \\
\hline 02 & $1495(7)$ & $5829(3)$ & $6675(3)$ & $27.3(17)$ \\
\hline 03 & 6903(6) & $7979(3)$ & $6653(3)$ & $30.3(18)$ \\
\hline 06 & 2694(7) & $9188(3)$ & $5776(3)$ & $36(2)$ \\
\hline F3 & $-1214(6)$ & $5574(4)$ & $5739(3)$ & $57(2)$ \\
\hline F7 & $3836(7)$ & $5153(4)$ & $6608(3)$ & $52.4(19)$ \\
\hline F4 & $26(7)$ & $6208(4)$ & $5903(3)$ & $56(2)$ \\
\hline F8 & $3568(7)$ & $4244(4)$ & $6807(3)$ & $56(2)$ \\
\hline C24 & $1117(9)$ & $7527(4)$ & $6362(4)$ & $21(2)$ \\
\hline C5 & $5264(8)$ & $7870(4)$ & $6428(3)$ & $18.4(18)$ \\
\hline C1 & 4034(8) & $7242(4)$ & $6694(3)$ & $16.3(15)$ \\
\hline $\mathrm{C} 2$ & $4774(8)$ & $7065(5)$ & $6977(3)$ & $18.8(17)$ \\
\hline C12 & 2761(8) & $8613(4)$ & $5722(4)$ & $25(2)$ \\
\hline C31 & 2898(9) & $6459(5)$ & $5298(4)$ & $19.8(15)$ \\
\hline $\mathrm{C} 45$ & 4993(9) & $6070(5)$ & $5927(4)$ & $20(2)$ \\
\hline C33 & 3597(9) & $6197(5)$ & $5038(3)$ & $22(2)$ \\
\hline C36 & $1506(9)$ & 6151(5) & $4864(4)$ & $23(2)$ \\
\hline C11 & $2373(11)$ & $8298(4)$ & $5423(4)$ & $31(3)$ \\
\hline C15 & $2551(10)$ & $6661(4)$ & $7203(3)$ & $19.6(15)$ \\
\hline $\mathrm{C} 20$ & $3114(8)$ & $6185(4)$ & $7312(3)$ & $17.9(17)$ \\
\hline C13 & $3367(9)$ & $8394(4)$ & $6031(4)$ & $21.6(19)$ \\
\hline C34 & $3281(10)$ & $5915(5)$ & $4694(4)$ & $26(2)$ \\
\hline $\mathrm{C} 4$ & $5990(8)$ & $7684(4)$ & $6707(4)$ & $20.5(19)$ \\
\hline C40 & 4712(8) & $6605(4)$ & $5809(3)$ & $18.2(16)$ \\
\hline C9 & $3180(9)$ & $7493(4)$ & $5758(4)$ & $17.9(16)$ \\
\hline C10 & 2591(10) & $7719(4)$ & $5448(3)$ & $23(2)$ \\
\hline C16 & $1854(9)$ & $6873(5)$ & $7478(3)$ & $21(2)$ \\
\hline $\mathrm{C} 8$ & $3597(8)$ & $7847(4)$ & $6070(3)$ & $17.4(14)$ \\
\hline C7 & $6651(9)$ & $8426(5)$ & $6390(4)$ & $31(3)$ \\
\hline C38 & $4047(10)$ & $5596(5)$ & $4437(4)$ & $32(3)$ \\
\hline C43 & $6754(9)$ & 6324(5) & $5836(4)$ & $28(2)$ \\
\hline C19 & $3025(9)$ & $5959(5)$ & $7700(4)$ & $20.6(17)$ \\
\hline C28 & $2006(9)$ & $8006(5)$ & $6895(4)$ & $28(2)$ \\
\hline C46 & 7301(9) & $7300(5)$ & $5649(4)$ & $33(3)$ \\
\hline C25 & $466(9)$ & $7972(5)$ & $6304(5)$ & $34(3)$ \\
\hline C39 & $362(10)$ & $6116(6)$ & $4771(4)$ & $34(3)$ \\
\hline C37 & $1862(9)$ & $6423(4)$ & $5205(4)$ & $21(2)$ \\
\hline C44 & $6044(9)$ & 5915(5) & $5927(4)$ & $28(2)$ \\
\hline $\mathrm{C} 23$ & 1882(9) & $7537(4)$ & $6651(4)$ & $19.9(15)$ \\
\hline C6 & $4289(8)$ & $7653(5)$ & $6398(3)$ & $16.9(16)$ \\
\hline C3 & $5779(9)$ & 7287(5) & $6981(4)$ & $29(3)$ \\
\hline C17 & $1710(10)$ & $6652(5)$ & $7855(4)$ & $29(2)$ \\
\hline C18 & $2298(9)$ & $6196(5)$ & $7965(3)$ & $30(2)$ \\
\hline C22 & $3639(11)$ & $5460(5)$ & $7822(4)$ & $35(3)$ \\
\hline C35 & $2232(9)$ & $5890(5)$ & $4613(4)$ & $32(3)$ \\
\hline C41 & $5451(8)$ & $6994(5)$ & $5697(3)$ & $21(2)$ \\
\hline $\mathrm{C} 27$ & 1338(10) & $8464(5)$ & $6846(5)$ & $37(3)$ \\
\hline C47 & $6362(10)$ & $5341(5)$ & $6036(5)$ & $36(3)$ \\
\hline C42 & $6511(9)$ & $6868(5)$ & $5728(4)$ & $26(2)$ \\
\hline C26 & 601(10) & $8440(5)$ & $6551(5)$ & $37(3)$ \\
\hline C29 & $-322(12)$ & $7986(6)$ & $5963(5)$ & $53(4)$ \\
\hline C21 & $920(11)$ & $6893(6)$ & $8148(4)$ & $42(3)$ \\
\hline C30 & $1465(13)$ & $8958(6)$ & $7128(6)$ & $57(4)$ \\
\hline C14 & $3269(14)$ & $9313(5)$ & $6128(5)$ & $54(5)$ \\
\hline B1 & $-215(14)$ & $5646(7)$ & $5884(6)$ & $48.5(19)$ \\
\hline B2 & $3111(14)$ & $4742(7)$ & $6703(6)$ & $42.2(17)$ \\
\hline
\end{tabular}


Table 2: Anisotropic Displacement Parameters $\left(\times 10^{4}\right) \mathrm{KWV}-\mathrm{II}-79$. The anisotropic displacement factor exponent takes the form: $-2 \pi^{2}\left[a^{* 2} \times U_{11}+\ldots 2 h k a^{*} \times b^{*} \times U_{12}\right]$

\begin{tabular}{|c|c|c|c|c|c|c|}
\hline Atom & $\mathrm{U}_{11}$ & $\mathrm{U}_{22}$ & $\mathrm{U}_{33}$ & $\mathrm{U}_{23}$ & $\mathrm{U}_{13}$ & $\mathrm{U}_{12}$ \\
\hline$\overline{\mathrm{Pd} 1}$ & $18.3(4)$ & $14.5(3)$ & $23.8(4)$ & $-2.0(3)$ & $1.4(4)$ & $-1.9(4)$ \\
\hline P2 & $14.2(12)$ & $11.9(11)$ & $22.9(12)$ & $-0.4(9)$ & $1.3(10)$ & $0.8(9)$ \\
\hline P1 & $13.3(12)$ & $10.6(10)$ & $23.3(12)$ & $-2.2(9)$ & $1.4(9)$ & $-0.3(8)$ \\
\hline 01 & $17(4)$ & $15(2)$ & $30(3)$ & $-5(2)$ & $3(3)$ & $-5(3)$ \\
\hline F6 & $52(3)$ & $25(3)$ & $44(3)$ & $1(2)$ & $7(3)$ & $4(3)$ \\
\hline 04 & $15(3)$ & $18(3)$ & $41(3)$ & $8(3)$ & $-3(2)$ & $-5(2)$ \\
\hline F2 & $27(3)$ & $34(4)$ & $73(4)$ & $-6(3)$ & $-3(3)$ & $-4(3)$ \\
\hline F5 & $51(3)$ & $29(3)$ & $45(3)$ & $0(2)$ & $7(3)$ & $4(3)$ \\
\hline 05 & $29(4)$ & $12(2)$ & $46(3)$ & $-1(2)$ & $-6(3)$ & $4(2)$ \\
\hline F1 & $44(4)$ & $44(4)$ & $78(4)$ & $0(3)$ & $-1(3)$ & $-17(3)$ \\
\hline 02 & $32(4)$ & $16(3)$ & $34(3)$ & $-2(2)$ & $9(3)$ & $-5(3)$ \\
\hline 03 & $18(2)$ & $24(3)$ & $48(4)$ & $11(3)$ & $-6(2)$ & $-6(2)$ \\
\hline 06 & $40(5)$ & $17(2)$ & $52(4)$ & $0(2)$ & $-11(4)$ & $5(2)$ \\
\hline F3 & $35(3)$ & $50(4)$ & $84(5)$ & $-5(4)$ & $3(3)$ & $2(3)$ \\
\hline F7 & $54(4)$ & $49(3)$ & $53(4)$ & $1(3)$ & $5(3)$ & $-4(3)$ \\
\hline F4 & $53(4)$ & $37(3)$ & $78(5)$ & $-7(3)$ & $20(3)$ & $-5(3)$ \\
\hline F8 & $67(4)$ & $42(3)$ & $60(4)$ & $3(3)$ & $7(3)$ & $17(3)$ \\
\hline C24 & $17(3)$ & $13(3)$ & $33(4)$ & $4(3)$ & $3(3)$ & $1(2)$ \\
\hline C5 & $15(2)$ & $8(3)$ & $32(3)$ & $0(3)$ & $0.3(19)$ & $-1.2(19)$ \\
\hline $\mathrm{C} 1$ & $15.2(19)$ & $10(3)$ & $24(3)$ & $-3(2)$ & $2.2(16)$ & $-0.3(16)$ \\
\hline $\mathrm{C} 2$ & $18(2)$ & $15(3)$ & $24(3)$ & $-1(2)$ & $0.1(17)$ & $-0.7(17)$ \\
\hline C12 & $20(5)$ & $17(2)$ & $40(3)$ & $2.2(18)$ & $-1(3)$ & $2(2)$ \\
\hline C31 & $20(2)$ & $16(3)$ & $23(2)$ & $-1.1(17)$ & $0.1(15)$ & $1.6(17)$ \\
\hline C45 & $19(3)$ & $16(3)$ & $25(6)$ & $-4(3)$ & $-2(2)$ & $2.9(19)$ \\
\hline C33 & $21(3)$ & $20(5)$ & $25(3)$ & $-4(3)$ & $0(2)$ & $3(2)$ \\
\hline C36 & $26(3)$ & $18(5)$ & $24(3)$ & $1(3)$ & $-2(2)$ & $-2(2)$ \\
\hline C11 & $31(6)$ & $19(2)$ & $43(4)$ & $2(2)$ & $-8(4)$ & $3(2)$ \\
\hline C15 & $18(3)$ & $17(2)$ & $24(2)$ & $-0.5(17)$ & $1.8(19)$ & $0(2)$ \\
\hline $\mathrm{C} 20$ & $18(3)$ & $15(2)$ & $21(2)$ & $-1.8(16)$ & $0.5(17)$ & $-1.4(19)$ \\
\hline C13 & $15(4)$ & $12.8(18)$ & $37(3)$ & $1.4(16)$ & $2(3)$ & $0.1(17)$ \\
\hline C34 & $27(3)$ & $24(5)$ & $27(3)$ & $-6(3)$ & $0(2)$ & $0(2)$ \\
\hline $\mathrm{C} 4$ & $16(2)$ & $12(3)$ & $34(3)$ & $0(3)$ & $-1(2)$ & $-1(2)$ \\
\hline $\mathrm{C} 40$ & $15.7(19)$ & $17(3)$ & $22(4)$ & $-4(2)$ & $0.7(18)$ & $3.4(16)$ \\
\hline C9 & $14(3)$ & $13.7(18)$ & $26(3)$ & $0.0(15)$ & $2(2)$ & $0.6(16)$ \\
\hline C10 & $20(5)$ & $18(2)$ & $30(3)$ & $2.0(19)$ & $-1(3)$ & $1(2)$ \\
\hline C16 & $17(4)$ & $20(3)$ & $25(3)$ & $-4(2)$ & $1(2)$ & $-1(3)$ \\
\hline C8 & $14(2)$ & $14.2(17)$ & $24(2)$ & $0.2(14)$ & $3.2(18)$ & $0.2(14)$ \\
\hline $\mathrm{C} 7$ & $20(3)$ & $23(3)$ & $50(5)$ & $11(3)$ & $-9(3)$ & $-9(3)$ \\
\hline C38 & $28(4)$ & $31(6)$ & $37(5)$ & $-15(5)$ & $2(3)$ & $-2(4)$ \\
\hline $\mathrm{C} 43$ & $19(3)$ & $22(3)$ & $44(6)$ & $-7(3)$ & $1(3)$ & $4(2)$ \\
\hline C19 & $21(3)$ & $18(2)$ & $22(2)$ & $0.0(17)$ & $0.1(18)$ & $-4(2)$ \\
\hline $\mathrm{C} 28$ & $22(4)$ & $19(2)$ & $43(4)$ & $-7(3)$ & $4(3)$ & $3(2)$ \\
\hline C46 & $21(4)$ & $29(4)$ & $51(7)$ & $-5(4)$ & $4(4)$ & $-1(3)$ \\
\hline $\mathrm{C} 25$ & $24(4)$ & $17(3)$ & $61(5)$ & $3(3)$ & $-4(4)$ & $6(3)$ \\
\hline C39 & $26(3)$ & $41(7)$ & $34(5)$ & $-2(5)$ & $-3(3)$ & $-2(3)$ \\
\hline C37 & $20(2)$ & $17(5)$ & $25(3)$ & $0(3)$ & $-0.4(19)$ & $2(2)$ \\
\hline C44 & $20(3)$ & $21(3)$ & $43(7)$ & $-4(3)$ & $-2(3)$ & $5(2)$ \\
\hline $\mathrm{C} 23$ & $17(2)$ & $15.5(19)$ & $27(3)$ & $1.5(16)$ & $5.3(18)$ & $2.0(16)$ \\
\hline C6 & $15(2)$ & $10(3)$ & $26(3)$ & $-2(2)$ & $1.5(18)$ & $-1.2(18)$ \\
\hline C3 & $18(2)$ & $24(4)$ & $43(4)$ & $11(4)$ & $-6(2)$ & $-5(2)$ \\
\hline C17 & $29(4)$ & $32(4)$ & $26(3)$ & $1(3)$ & $4(3)$ & $4(4)$ \\
\hline C18 & $32(4)$ & $33(4)$ & $26(3)$ & $2(3)$ & $6(3)$ & $5(4)$ \\
\hline $\mathrm{C} 22$ & $41(6)$ & $31(4)$ & $32(5)$ & $8(4)$ & $7(4)$ & $11(4)$ \\
\hline C35 & $27(3)$ & $36(6)$ & $32(4)$ & $-11(4)$ & $-2(2)$ & $0(2)$ \\
\hline C41 & $18(2)$ & $17(3)$ & $28(5)$ & $-5(3)$ & $4(2)$ & $2.5(19)$ \\
\hline
\end{tabular}




\begin{tabular}{llllrrr}
\hline Atom & $\mathbf{U}_{11}$ & $\mathbf{U}_{22}$ & $\mathbf{U}_{33}$ & $\mathbf{U}_{23}$ & $\mathbf{U}_{13}$ & $\mathbf{U}_{12}$ \\
\hline C27 & $26(4)$ & $21(3)$ & $63(5)$ & $-6(3)$ & $2(4)$ & $7(3)$ \\
C47 & $26(5)$ & $22(3)$ & $62(8)$ & $-1(3)$ & $-9(5)$ & $5(3)$ \\
C42 & $17(3)$ & $22(3)$ & $39(6)$ & $-7(3)$ & $4(2)$ & $4(2)$ \\
C26 & $26(4)$ & $20(3)$ & $65(5)$ & $-1(3)$ & $0(4)$ & $3(3)$ \\
C29 & $49(6)$ & $25(6)$ & $83(7)$ & $0(5)$ & $-28(6)$ & $10(5)$ \\
C21 & $48(6)$ & $43(6)$ & $37(4)$ & $6(4)$ & $17(4)$ & $15(5)$ \\
C30 & $44(7)$ & $35(4)$ & $92(8)$ & $-26(5)$ & $-12(6)$ & $16(5)$ \\
C14 & $78(9)$ & $14(3)$ & $70(6)$ & $-4(2)$ & $-38(7)$ & $11(3)$ \\
B1 & $34(3)$ & $35(3)$ & $76(4)$ & $-3(3)$ & $5(3)$ & $0(3)$ \\
B2 & $47(3)$ & $35(3)$ & $45(3)$ & $-2(2)$ & $6(3)$ & $6(3)$
\end{tabular}

Table 3: Bond Lengths in Å for KWV-II-79.

\begin{tabular}{|c|c|c|}
\hline Atom & Atom & Length/Å \\
\hline Pd1 & P2 & $2.240(3)$ \\
\hline Pd1 & P1 & $2.225(3)$ \\
\hline Pd1 & 01 & $2.147(6)$ \\
\hline Pd1 & 02 & $2.157(8)$ \\
\hline P2 & C31 & $1.815(12)$ \\
\hline P2 & C40 & $1.805(11)$ \\
\hline P2 & C9 & $1.808(10)$ \\
\hline P1 & C1 & $1.807(11)$ \\
\hline P1 & C15 & $1.812(10)$ \\
\hline P1 & $\mathrm{C} 23$ & $1.803(11)$ \\
\hline F6 & B2 & $1.38(2)$ \\
\hline 04 & C5 & $1.394(13)$ \\
\hline 04 & C7 & $1.458(14)$ \\
\hline $\mathrm{F} 2$ & B1 & $1.38(2)$ \\
\hline F5 & B2 & $1.373(19)$ \\
\hline 05 & C13 & $1.393(14)$ \\
\hline 05 & C14 & $1.403(15)$ \\
\hline F1 & B1 & $1.39(2)$ \\
\hline 03 & C4 & $1.392(13)$ \\
\hline 03 & C7 & $1.416(14)$ \\
\hline 06 & C12 & $1.391(13)$ \\
\hline 06 & C14 & $1.410(16)$ \\
\hline F3 & B1 & $1.39(2)$ \\
\hline F7 & B2 & $1.40(2)$ \\
\hline F4 & B1 & $1.385(19)$ \\
\hline F8 & B2 & $1.375(19)$ \\
\hline C24 & $\mathrm{C} 25$ & $1.375(15)$ \\
\hline $\mathrm{C} 24$ & $\mathrm{C} 23$ & $1.374(17)$ \\
\hline C5 & $\mathrm{C} 4$ & $1.388(16)$ \\
\hline C5 & C6 & $1.373(15)$ \\
\hline C1 & $\mathrm{C} 2$ & $1.405(15)$ \\
\hline C1 & C6 & $1.422(15)$ \\
\hline $\mathrm{C} 2$ & C3 & $1.409(16)$ \\
\hline C12 & C11 & $1.338(16)$ \\
\hline C12 & C13 & $1.386(16)$ \\
\hline C31 & C33 & $1.396(16)$ \\
\hline C31 & C37 & $1.383(15)$ \\
\hline C45 & C40 & $1.387(16)$ \\
\hline C45 & C44 & $1.415(16)$ \\
\hline C33 & C34 & $1.381(16)$ \\
\hline C36 & C39 & $1.519(17)$ \\
\hline C36 & C37 & $1.378(16)$ \\
\hline C36 & C35 & $1.400(17)$ \\
\hline
\end{tabular}




\begin{tabular}{lll}
\hline Atom & Atom & Length/A \\
\hline C11 & C10 & $1.418(15)$ \\
C15 & C20 & $1.401(15)$ \\
C15 & C16 & $1.377(16)$ \\
C20 & C19 & $1.392(16)$ \\
C13 & C8 & $1.351(15)$ \\
C34 & C38 & $1.512(17)$ \\
C34 & C35 & $1.390(19)$ \\
C4 & C3 & $1.340(17)$ \\
C40 & C41 & $1.387(16)$ \\
C9 & C10 & $1.384(16)$ \\
C9 & C8 & $1.436(16)$ \\
C16 & C17 & $1.359(17)$ \\
C8 & C6 & $1.480(16)$ \\
C43 & C44 & $1.378(17)$ \\
C43 & C42 & $1.387(17)$ \\
C19 & C18 & $1.405(16)$ \\
C19 & C22 & $1.491(16)$ \\
C28 & C23 & $1.391(16)$ \\
C28 & C27 & $1.408(17)$ \\
C46 & C42 & $1.480(16)$ \\
C25 & C26 & $1.396(18)$ \\
C25 & C29 & $1.519(19)$ \\
C44 & C47 & $1.481(17)$ \\
C17 & C18 & $1.381(17)$ \\
C17 & C21 & $1.522(17)$ \\
C41 & C42 & $1.414(15)$ \\
C27 & C26 & $1.36(2)$ \\
C27 & C30 & $1.514(19)$ \\
& &
\end{tabular}

Table 4: Bond Angles in ${ }^{\circ}$ for KWV-II-79.

\begin{tabular}{llll}
\hline Atom & Atom & Atom & \multicolumn{1}{c}{ Angle $^{\circ}$} \\
\hline P1 & Pd1 & P2 & $90.46(11)$ \\
O1 & Pd1 & P2 & $91.2(2)$ \\
O1 & Pd1 & P1 & $171.0(2)$ \\
O1 & Pd1 & O2 & $87.3(3)$ \\
O2 & Pd1 & P2 & $174.6(3)$ \\
O2 & Pd1 & P1 & $91.8(2)$ \\
C31 & P2 & Pd1 & $104.5(4)$ \\
C40 & P2 & Pd1 & $111.6(4)$ \\
C40 & P2 & C31 & $107.1(5)$ \\
C40 & P2 & C9 & $107.8(5)$ \\
C9 & P2 & Pd1 & $117.7(4)$ \\
C9 & P2 & C31 & $107.6(5)$ \\
C1 & P1 & Pd1 & $115.0(4)$ \\
C1 & P1 & C15 & $106.9(6)$ \\
C15 & P1 & Pd1 & $107.3(3)$ \\
C23 & P1 & Pd1 & $114.0(4)$ \\
C23 & P1 & C1 & $106.1(5)$ \\
C23 & P1 & C15 & $107.1(5)$ \\
C5 & O4 & C7 & $103.7(8)$ \\
C13 & O5 & C14 & $105.6(9)$ \\
C4 & O3 & C7 & $105.3(8)$ \\
C12 & O6 & C14 & $106.3(9)$ \\
C23 & C24 & C25 & $121.8(11)$
\end{tabular}

\begin{tabular}{llll}
\hline Atom & Atom & Atom & \multicolumn{1}{c}{ Angle $^{\circ}$} \\
\hline C4 & C5 & O4 & $109.3(9)$ \\
C6 & C5 & O4 & $127.1(10)$ \\
C6 & C5 & C4 & $123.4(10)$ \\
C2 & C1 & P1 & $120.5(8)$ \\
C2 & C1 & C6 & $120.2(10)$ \\
C6 & C1 & P1 & $119.3(8)$ \\
C1 & C2 & C3 & $121.8(11)$ \\
C11 & C12 & O6 & $128.9(11)$ \\
C11 & C12 & C13 & $122.5(10)$ \\
C13 & C12 & O6 & $108.5(10)$ \\
C33 & C31 & P2 & $119.5(9)$ \\
C37 & C31 & P2 & $122.0(9)$ \\
C37 & C31 & C33 & $117.9(11)$ \\
C40 & C45 & C44 & $119.7(11)$ \\
C34 & C33 & C31 & $122.0(11)$ \\
C37 & C36 & C39 & $121.2(11)$ \\
C37 & C36 & C35 & $117.7(11)$ \\
C35 & C36 & C39 & $121.1(11)$ \\
C12 & C11 & C10 & $115.7(11)$ \\
C20 & C15 & P1 & $118.2(8)$ \\
C16 & C15 & P1 & $123.3(8)$ \\
C16 & C15 & C20 & $118.5(10)$ \\
C19 & C20 & C15 & $120.5(10)$
\end{tabular}




\begin{tabular}{|c|c|c|c|c|c|c|c|}
\hline Atom & Atom & Atom & Angle/ $/^{\circ}$ & Atom & Atom & Atom & Angle $/^{\circ}$ \\
\hline$\overline{\mathrm{C} 12}$ & C13 & 05 & $109.8(9)$ & $\overline{\mathrm{C} 24}$ & $\mathrm{C} 23$ & P1 & $120.0(9)$ \\
\hline C8 & C13 & 05 & $126.0(11)$ & $\mathrm{C} 24$ & $\mathrm{C} 23$ & $\mathrm{C} 28$ & $119.8(11)$ \\
\hline C8 & C13 & C12 & $124.2(11)$ & $\mathrm{C} 28$ & $\mathrm{C} 23$ & P1 & $120.2(9)$ \\
\hline C33 & C34 & C38 & $120.7(11)$ & $\mathrm{C} 5$ & C6 & $\mathrm{C} 1$ & $115.4(10)$ \\
\hline C33 & C34 & C35 & $118.0(12)$ & $\mathrm{C} 5$ & C6 & $\mathrm{C} 8$ & $119.5(10)$ \\
\hline C35 & C34 & C38 & 121.1(11) & $\mathrm{C} 1$ & C6 & C8 & $125.1(10)$ \\
\hline C5 & $\mathrm{C} 4$ & 03 & $109.4(9)$ & $\mathrm{C} 4$ & C3 & $\mathrm{C} 2$ & $116.8(11)$ \\
\hline C3 & $\mathrm{C} 4$ & 03 & $128.3(11)$ & C16 & C17 & C18 & $118.2(11)$ \\
\hline C3 & $\mathrm{C} 4$ & $\mathrm{C} 5$ & $122.3(11)$ & C16 & C17 & $\mathrm{C} 21$ & $121.3(11)$ \\
\hline C45 & $\mathrm{C} 40$ & P2 & $116.4(9)$ & C18 & C17 & C21 & $120.5(11)$ \\
\hline C45 & $\mathrm{C} 40$ & C41 & $120.9(10)$ & C17 & C18 & C19 & $121.9(11)$ \\
\hline C41 & $\mathrm{C} 40$ & P2 & $122.6(8)$ & C34 & C35 & C36 & $121.9(12)$ \\
\hline C10 & C9 & P2 & 118.8(8) & C40 & C41 & C42 & $120.7(11)$ \\
\hline C10 & C9 & $\mathrm{C} 8$ & $120.2(9)$ & $\mathrm{C} 28$ & $\mathrm{C} 27$ & $\mathrm{C} 30$ & $118.2(13)$ \\
\hline $\mathrm{C} 8$ & C9 & P2 & $120.9(8)$ & $\mathrm{C} 26$ & $\mathrm{C} 27$ & $\mathrm{C} 28$ & $118.8(12)$ \\
\hline $\mathrm{C} 9$ & $\mathrm{C} 10$ & C11 & $122.4(11)$ & $\mathrm{C} 26$ & $\mathrm{C} 27$ & C30 & $123.0(12)$ \\
\hline C17 & C16 & C15 & $122.9(11)$ & C43 & $\mathrm{C} 42$ & C46 & $123.0(11)$ \\
\hline C13 & $\mathrm{C} 8$ & $\mathrm{C} 9$ & $115.0(10)$ & $\mathrm{C} 43$ & $\mathrm{C} 42$ & C41 & $116.1(11)$ \\
\hline C13 & C8 & C6 & $120.6(10)$ & C41 & C42 & C46 & $120.9(10)$ \\
\hline C9 & C8 & C6 & $124.3(10)$ & $\mathrm{C} 27$ & $\mathrm{C} 26$ & $\mathrm{C} 25$ & $122.4(12)$ \\
\hline 03 & $\mathrm{C} 7$ & 04 & 107.1(9) & 05 & C14 & 06 & $109.7(10)$ \\
\hline C44 & $\mathrm{C} 43$ & $\mathrm{C} 42$ & $124.9(11)$ & F2 & B1 & F1 & $109.7(14)$ \\
\hline $\mathrm{C} 20$ & C19 & C18 & $117.8(10)$ & F2 & B1 & F3 & $108.6(14)$ \\
\hline $\mathrm{C} 20$ & C19 & $\mathrm{C} 22$ & $120.9(10)$ & F2 & B1 & F4 & $111.0(14)$ \\
\hline C18 & C19 & $\mathrm{C} 22$ & $121.2(11)$ & F1 & B1 & F3 & $108.9(14)$ \\
\hline $\mathrm{C} 23$ & $\mathrm{C} 28$ & $\mathrm{C} 27$ & $119.5(12)$ & F4 & B1 & F1 & $108.4(15)$ \\
\hline C24 & $\mathrm{C} 25$ & $\mathrm{C} 26$ & $117.8(12)$ & F4 & B1 & F3 & $110.2(14)$ \\
\hline $\mathrm{C} 24$ & $\mathrm{C} 25$ & $\mathrm{C} 29$ & $122.3(12)$ & F6 & B2 & F7 & $107.9(13)$ \\
\hline $\mathrm{C} 26$ & $\mathrm{C} 25$ & $\mathrm{C} 29$ & $119.8(11)$ & F5 & B2 & F6 & $109.6(13)$ \\
\hline C36 & $\mathrm{C} 37$ & C31 & $122.5(11)$ & F5 & B2 & F7 & $108.5(13)$ \\
\hline C45 & $\mathrm{C} 44$ & $\mathrm{C} 47$ & $120.9(11)$ & F5 & B2 & F8 & $110.2(13)$ \\
\hline $\mathrm{C} 43$ & $\mathrm{C} 44$ & C45 & $117.3(11)$ & F8 & B2 & F6 & $108.6(13)$ \\
\hline C43 & $\mathrm{C} 44$ & C47 & $121.8(11)$ & F8 & B2 & F7 & $112.0(13)$ \\
\hline
\end{tabular}

Table 5: Hydrogen Fractional Atomic Coordinates $(\times 104)$ and Equivalent Isotropic Displacement Parameters $\left(\AA^{2} \times 10^{3}\right)$ for KWV-II-79. $U_{e q}$ is defined as $1 / 3$ of of the trace of the orthogonalised $U_{i j .}$

\begin{tabular}{|c|c|c|c|c|}
\hline Atom & $x$ & $y$ & $\mathrm{z}$ & $U_{e q}$ \\
\hline$\overline{\mathrm{H} 1 \mathrm{~A}}$ & $1820(30)$ & $5470(50)$ & $5680(16)$ & 31 \\
\hline H1B & $2370(80)$ & $5300(20)$ & $6095(17)$ & 31 \\
\hline $\mathrm{H} 2 \mathrm{~A}$ & $1080(40)$ & $5640(40)$ & $6470(20)$ & 41 \\
\hline $\mathrm{H} 2 \mathrm{~B}$ & $1740(70)$ & $5510(30)$ & $6850(20)$ & 41 \\
\hline $\mathrm{H} 24$ & 1037 & 7204 & 6198 & 25 \\
\hline $\mathrm{H} 2$ & 4593 & 6788 & 7171 & 23 \\
\hline $\mathrm{H} 45$ & 4481 & 5809 & 6007 & 24 \\
\hline H33 & 4311 & 6214 & 5099 & 27 \\
\hline H11 & 1977 & 8452 & 5207 & 37 \\
\hline $\mathrm{H} 20$ & 3559 & 6015 & 7119 & 21 \\
\hline H10 & 2323 & 7478 & 5244 & 27 \\
\hline H16 & 1457 & 7189 & 7402 & 25 \\
\hline H7A & 7218 & 8492 & 6194 & 37 \\
\hline H7B & 6538 & 8772 & 6548 & 37 \\
\hline H38A & 4748 & 5712 & 4508 & 48 \\
\hline H38B & 3921 & 5674 & 4148 & 48 \\
\hline H38C & 3969 & 5195 & 4487 & 48 \\
\hline
\end{tabular}




\begin{tabular}{|c|c|c|c|c|c|}
\hline Atom & & & & & $U_{e q}$ \\
\hline$\overline{\mathrm{H} 43}$ & 7462 & 6225 & 5847 & 34 & \\
\hline H28 & 2537 & 8018 & 7094 & 33 & \\
\hline $\mathrm{H} 46 \mathrm{~A}$ & 7589 & 7430 & 5908 & 50 & \\
\hline $\mathrm{H} 46 \mathrm{~B}$ & 6986 & 7614 & 5505 & 50 & \\
\hline $\mathrm{H} 46 \mathrm{C}$ & 7852 & 7141 & 5481 & 50 & \\
\hline H39A & 11 & 5916 & 4991 & 51 & \\
\hline H39B & 259 & 5915 & 4514 & 51 & \\
\hline H39C & 78 & 6493 & 4747 & 51 & \\
\hline H37 & 1377 & 6592 & 5383 & 25 & \\
\hline H3 & 6282 & 7161 & 7170 & 34 & \\
\hline H18 & 2209 & 6039 & 8228 & 36 & \\
\hline $\mathrm{H} 22 \mathrm{~A}$ & 3983 & 5304 & 7582 & 52 & \\
\hline H22B & 3181 & 5178 & 7940 & 52 & \\
\hline $\mathrm{H} 22 \mathrm{C}$ & 4158 & 5570 & 8023 & 52 & \\
\hline H35 & 2001 & 5690 & 4380 & 38 & \\
\hline H41 & 5241 & 7349 & 5600 & 25 & \\
\hline $\mathrm{H} 47 \mathrm{~A}$ & 5798 & 5153 & 6180 & 55 & \\
\hline H47B & 6970 & 5356 & 6213 & 55 & \\
\hline $\mathrm{H} 47 \mathrm{C}$ & 6529 & 5132 & 5788 & 55 & \\
\hline H26 & 163 & 8753 & 6513 & 44 & \\
\hline H29A & -48 & 8203 & 5734 & 79 & \\
\hline H29B & -958 & 8161 & 6061 & 79 & \\
\hline $\mathrm{H} 29 \mathrm{C}$ & -469 & 7605 & 5873 & 79 & \\
\hline $\mathrm{H} 21 \mathrm{~A}$ & 844 & 7294 & 8097 & 64 & \\
\hline H21B & 1150 & 6833 & 8428 & 64 & \\
\hline $\mathrm{H} 21 \mathrm{C}$ & 255 & 6708 & 8106 & 64 & \\
\hline H30A & 1297 & 8845 & 7407 & 85 & \\
\hline H30B & 1000 & 9259 & 7044 & 85 & \\
\hline H30C & 2178 & 9091 & 7118 & 85 & \\
\hline $\mathrm{H} 14 \mathrm{~A}$ & 3833 & 9574 & 6059 & 65 & \\
\hline H14B & 2820 & 9495 & 6332 & 65 & \\
\hline
\end{tabular}

Table 6: Hydrogen Bond information for KWV-II-79.

\begin{tabular}{|c|c|c|c|c|c|c|}
\hline D & $\mathrm{H}$ & A & $\mathrm{d}(\mathrm{D}-\mathrm{H}) / \AA$ & $\mathrm{d}(\mathrm{H}-\mathrm{A}) / \AA$ & $\mathrm{d}(\mathrm{D}-\mathrm{A}) / \AA$ & D-H-A/deg \\
\hline 01 & $\mathrm{H} 1 \mathrm{~A}$ & F2 & $0.99(3)$ & $1.80(3)$ & $2.674(11)$ & $146(5)$ \\
\hline 01 & H1B & F6 & $0.96(2)$ & $1.78(3)$ & $2.689(9)$ & $157(7)$ \\
\hline 02 & $\mathrm{H} 2 \mathrm{~A}$ & $\mathrm{~F} 1$ & $0.98(3)$ & $1.79(3)$ & $2.693(12)$ & 151(6) \\
\hline 02 & $\mathrm{H} 2 \mathrm{~B}$ & F5 & $1.01(3)$ & $1.80(3)$ & $2.765(11)$ & $159(8)$ \\
\hline
\end{tabular}


Submitted by: Jeremy Morgan

Solved by: John Bacsa

$R_{1}=3.46 \%$

Sample ID: KWV-II-172

\section{Crystal Data and Experimental}

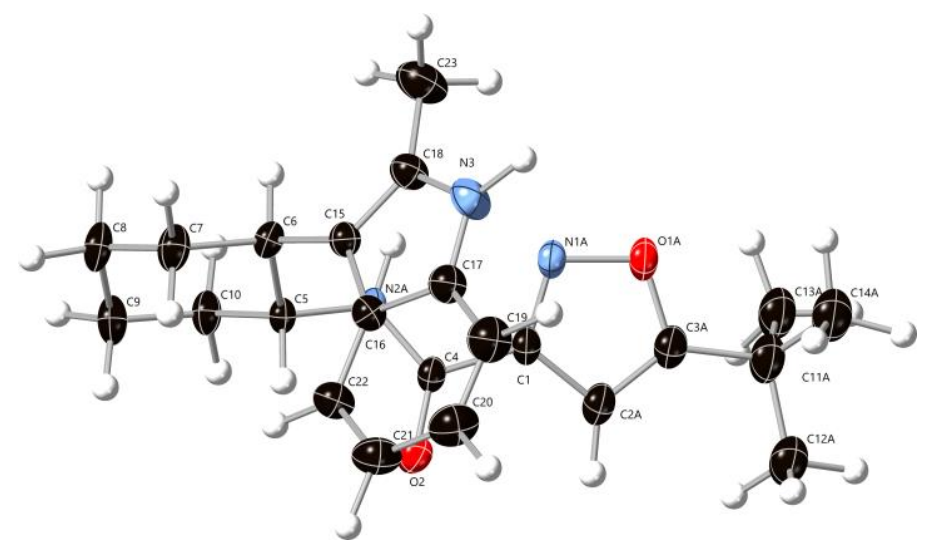

Experimental. Single colorless needle-shaped crystals of KWV-II-172 recrystallized from DCM by slow evaporation. A suitable crystal with dimensions $1.37 \times 0.71 \times 0.39 \mathrm{~mm}^{3}$ was selected and mounted on a loop with paratone oil on a Bruker APEX-II CCD diffractometer. The crystal was kept at a steady $T=$ 173(2) K during data collection. The structure was solved with the ShelXT 2014/4 (Sheldrick, 2014) solution program using dual methods and by using 0lex2 1.3-alpha (Dolomanov et al., 2009) as the graphical interface. The model was refined with olex2.refine 1.3-alpha (Bourhis et al., 2015) using full matrix least squares minimisation on $F^{2}$.

Crystal Data. $\quad \mathrm{C}_{23} \mathrm{H}_{29} \mathrm{~N}_{3} \mathrm{O}_{2}, M_{r}=379.506$, monoclinic, $C 2$ (No. 5), $\mathrm{a}=18.6878(7) \AA, \quad \mathrm{b}=9.7545(4) \AA, \quad \mathrm{c}=$ 14.4741(5) $\AA, \quad \beta=125.282(1)^{\circ}, \quad \alpha=\gamma=90^{\circ}, \quad V=$ 2153.85(14) $\AA^{3}, T=173(2) \mathrm{K}, Z=4, Z^{\prime}=1, \mu\left(\mathrm{CuK}_{\alpha}\right)=0.597$, 7814 reflections measured, 3430 unique $\left(\mathrm{R}_{\text {int }}=0.0221\right)$ which were used in all calculations. The final $w R_{2}$ was 0.0913 (all data) and $R_{1}$ was 0.0346 (I $\geq 2 \sigma(\mathrm{I})$ ).

\begin{tabular}{|c|c|}
\hline Compound & KWV-II-172 \\
\hline Formula & $\mathrm{C}_{23} \mathrm{H}_{29} \mathrm{~N}_{3} \mathrm{O}_{2}$ \\
\hline$D_{\text {calc. }} / \mathrm{g} \mathrm{cm}^{-3}$ & 1.170 \\
\hline$\mu / \mathrm{mm}^{-1}$ & 0.597 \\
\hline Formula Weight & 379.506 \\
\hline Color & colorless \\
\hline Shape & needle-shaped \\
\hline Size $/ \mathrm{mm}^{3}$ & $1.37 \times 0.71 \times 0.39$ \\
\hline$T / K^{\prime}$ & $173(2)$ \\
\hline Crystal System & monoclinic \\
\hline Flack Parameter & $-0.1(3)$ \\
\hline Hooft Parameter & $0.06(6)$ \\
\hline Space Group & $C 2$ \\
\hline$a / \AA ̊$ & $18.6878(7)$ \\
\hline$b / \AA$ & $9.7545(4)$ \\
\hline$c / \AA ̊$ & $14.4741(5)$ \\
\hline$\alpha /^{\circ}$ & 90 \\
\hline$\beta /^{\circ}$ & $125.282(1)$ \\
\hline$\left.\gamma\right|^{\circ}$ & 90 \\
\hline $\mathrm{V} / \AA^{3}$ & $2153.85(14)$ \\
\hline$Z$ & 4 \\
\hline$Z^{\prime}$ & 1 \\
\hline Wavelength/Å & 1.54178 \\
\hline Radiation type & $\mathrm{CuK}_{\alpha}$ \\
\hline$\Theta_{\min } /^{\circ}$ & 3.74 \\
\hline$\left.\Theta_{\max }\right|^{\circ}$ & 68.28 \\
\hline Measured Refl's. & 7814 \\
\hline Indep't Refl's & 3430 \\
\hline Refl's $I \geq 2 \sigma(I)$ & 3404 \\
\hline$R_{\text {int }}$ & 0.0221 \\
\hline Parameters & 351 \\
\hline Restraints & 300 \\
\hline Largest Peak & 0.2831 \\
\hline Deepest Hole & -0.2827 \\
\hline GooF & 1.0596 \\
\hline$w R_{2}$ (all data) & 0.0913 \\
\hline$w R_{2}$ & 0.0912 \\
\hline$R_{1}$ (all data) & 0.0348 \\
\hline$R_{1}$ & 0.0346 \\
\hline
\end{tabular}




\section{Structure Quality Indicators}
Reflections:
${ }_{2 \theta=136.6^{\circ}}^{d} \min ($ Cula $) 0.83$
$1 / \sigma(\mathrm{I})$
42.1
Rint
$2.21 \%$
Full $135.4^{\circ} .99 .5$
$99 \%$ to 136.6
Refinement:
Shift 0.001
Max Peak 0.3
Min Peak
$-0.3$
Goof 1.060 Flack
$-.1(3)$

A colourless needle-shaped-shaped crystal with dimensions $1.37 \times 0.71 \times 0.39 \mathrm{~mm}^{3}$ was mounted on a loop with paratone oil. Data were collected using a Bruker APEX-II CCD diffractometer equipped with an Oxford Cryosystems low-temperature device operating at $T=173(2) \mathrm{K}$.

Data were measured using $\phi$ and $\omega$ scans using $\mathrm{CuK}_{\alpha}$ radiation. The diffraction pattern was indexed and the total number of runs and images was based on the strategy calculation from the program APEX2 v2014.11 (Bruker, 2014). The maximum resolution that was achieved was $\Theta=68.28^{\circ}(0.83 \AA)$.

The unit cell was refined using SAINT v8.34A (Bruker, 2013) on 7566 reflections, 97\% of the observed reflections.

Data reduction, scaling and absorption corrections were performed using SAINT v8.34A (Bruker, 2013). The final completeness is $99.57 \%$ out to $68.28^{\circ}$ in $\Theta$. SADABS-2014/5 (Bruker, 2014/5) was used for absorption correction. $w R_{2}$ (int) was 0.1149 before and 0.0290 after correction. The Ratio of minimum to maximum transmission is 0.7642 . The $\lambda / 2$ correction factor is 0.00150 . The absorption coefficient $\mu$ of this material is $0.597 \mathrm{~mm}^{-1}$ at this wavelength $(\lambda=1.54178 \AA)$ and the minimum and maximum transmissions are 0.576 and 0.753 .

The structure was solved and the space group C2 (\# 5) determined by the ShelXT 2014/4 (Sheldrick, 2014) structure solution program using using dual methods and refined by full matrix least squares minimisation on $\boldsymbol{F}^{2}$ using version of olex2.refine 1.3-alpha (Bourhis et al., 2015). All non-hydrogen atoms were refined anisotropically. Hydrogen atom positions were calculated geometrically and refined using the riding model. Most hydrogen atom positions were calculated geometrically and refined using the riding model, but some hydrogen atoms were refined freely.

_refine_special_details: Refinement using NoSpherA2, an implementation of NOn-SPHERical Atom-formfactors in Olex2.Please cite:F. Kleemiss et al. DOI 10.1039/D0SC05526C - 2020NoSpherA2 implementation of HAR makes use of tailor-made aspherical atomic form factors calculatedon-the-fly from a Hirshfeldpartitioned electron density (ED) - not fromspherical-atom form factors.The ED is calculated from a gaussian basis set single determinant SCFwavefunction - either Hartree-Fock or DFT using selected funtionals - for a fragment of the crystal.This fregment can be embedded in an electrostatic crystal field by employing cluster charges.The following options were used: SOFTWARE: ORCA PARTITIONING: NoSpherA2 INT ACCURACY: Normal METHOD: PBE BASIS SET: def2-SVP CHARGE: 0 MULTIPLICITY: 1 DATE: 2021-07-23_14-53-51

The Flack parameter was refined to 0.01(6), confirming the absolute stereochemistry. Determination of absolute structure using Bayesian statistics on Bijvoet differences using the program within Platon (Spek, 2003) also report that we have the correct enantiomer based on this comparison. Note: The Flack parameter is used to determine chirality of the crystal studied, the value should be near 0 , a value of 1 mans that the stereochemistry is wrong and the model should be inverted. A value of 0.5 means that the crystal consists of a racemic mixture of the two enantiomers. 


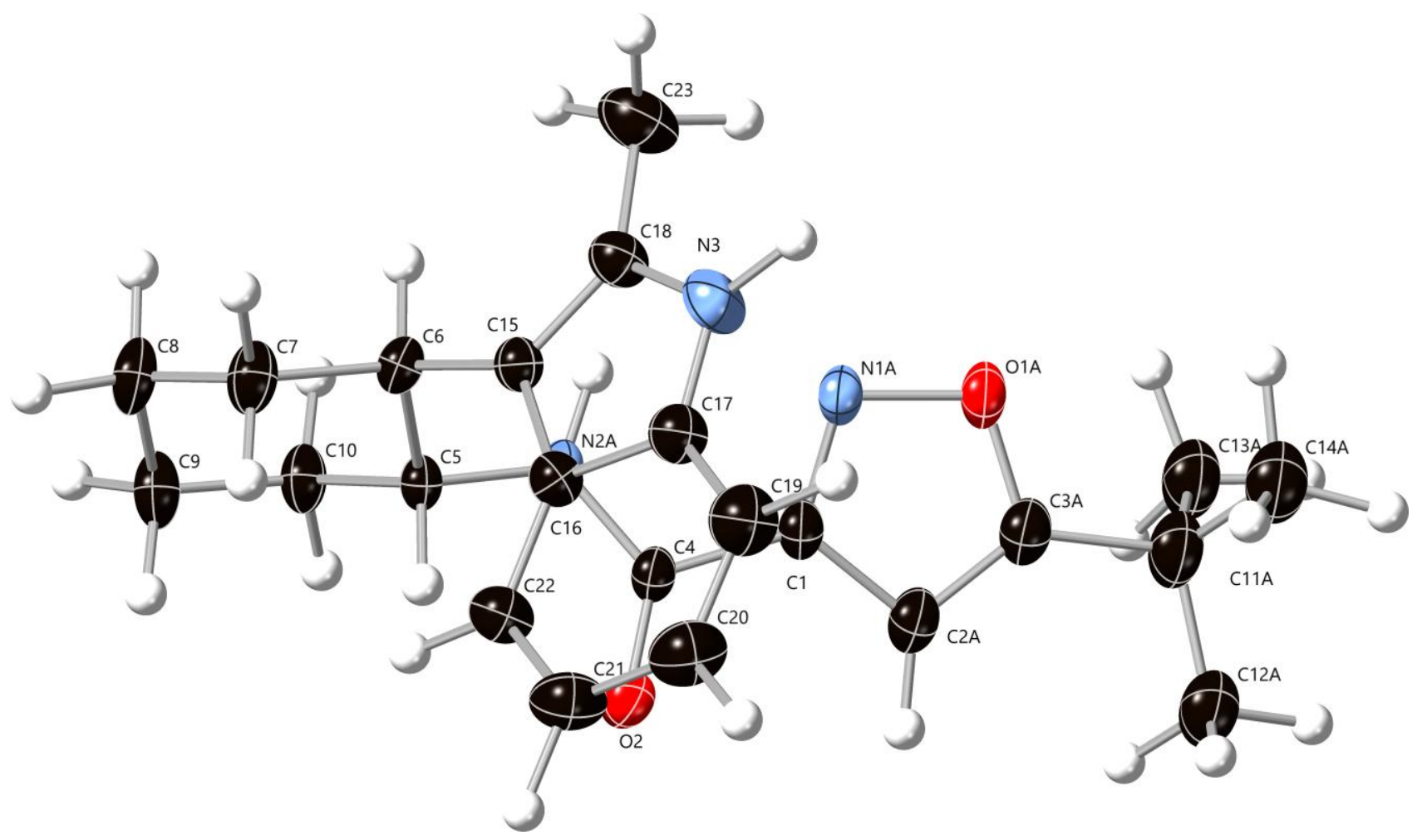

Figure 1: Representation of the asymmetric unit with thermal ellipsoids at 50\% probability level.

\section{Data Plots: Diffraction Data}
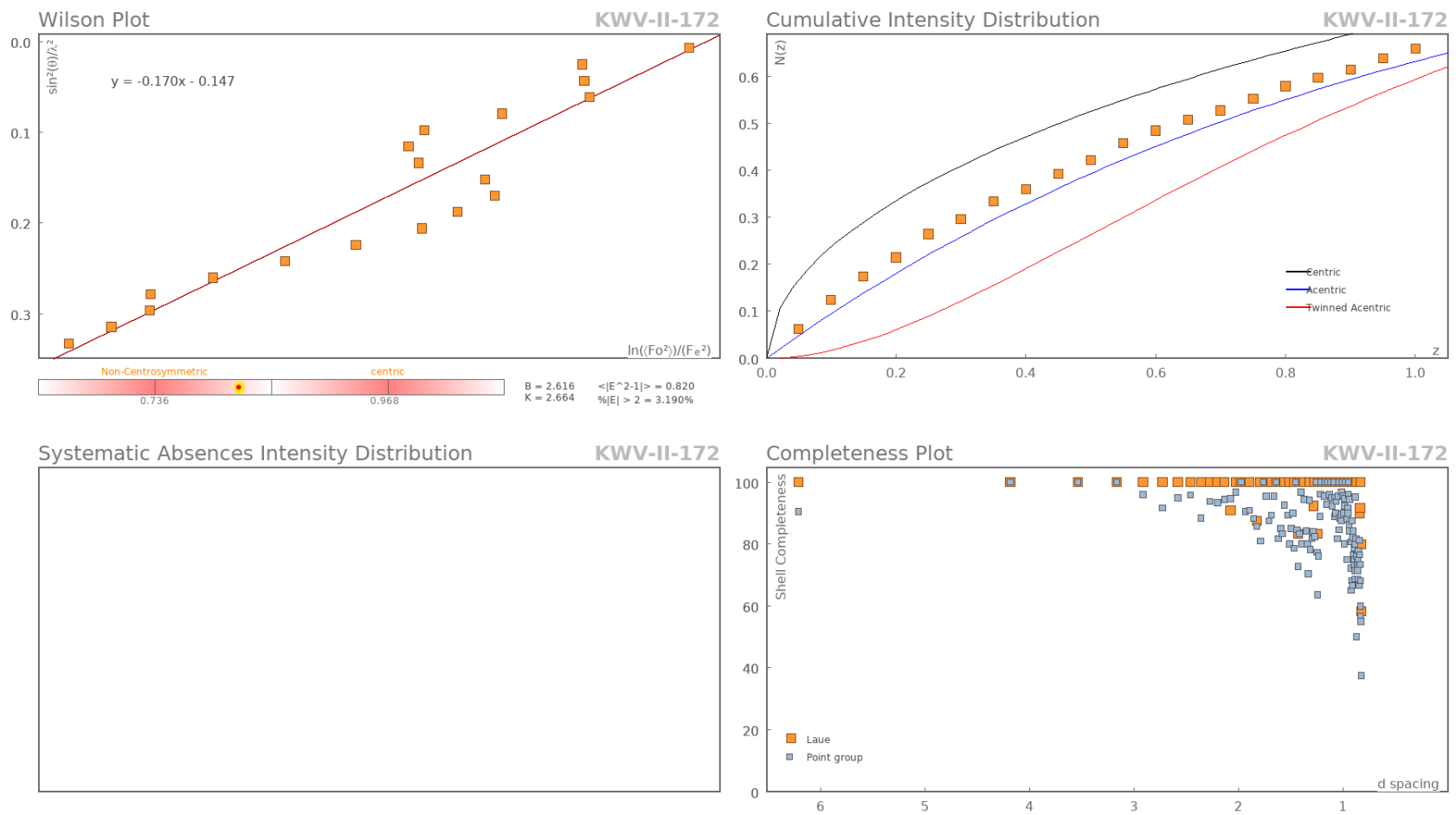


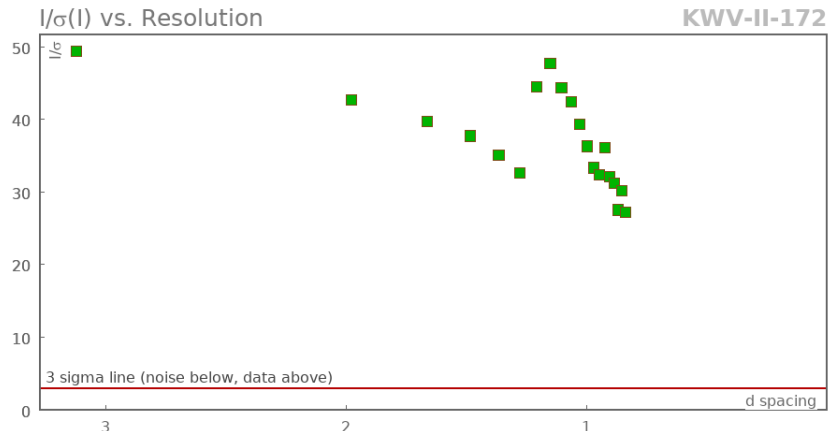

\section{Data Plots: Refinement and Data}
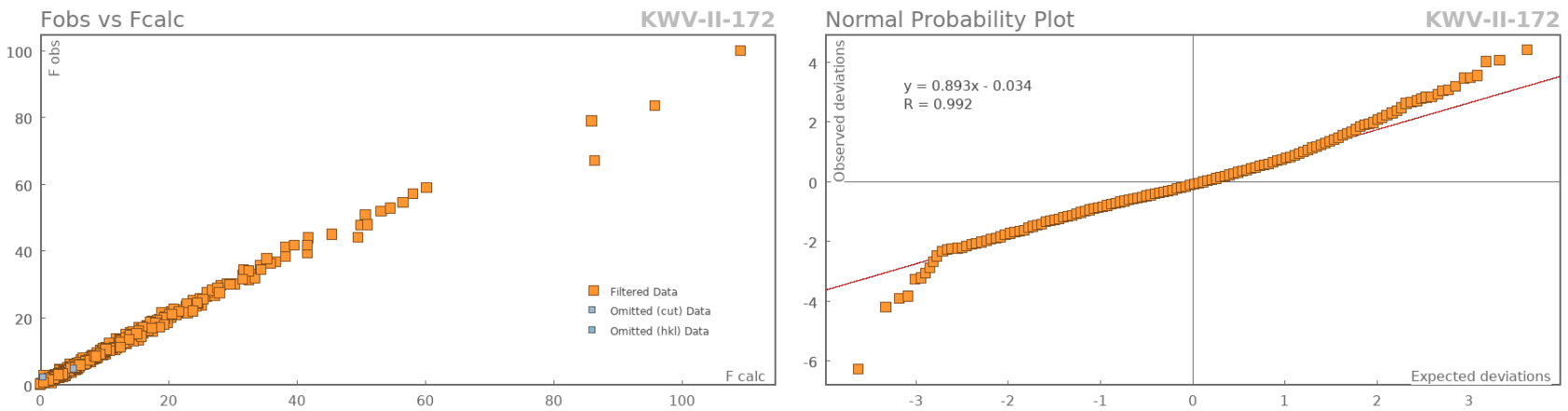

Table 1: Fractional Atomic Coordinates $\left(\times 10^{4}\right)$ and Equivalent Isotropic Displacement Parameters $\left(\AA^{2} \times 10^{3}\right)$ for KWV-II-172. $U_{e q}$ is defined as $1 / 3$ of the trace of the orthogonalised $U_{i j}$.

\begin{tabular}{lllll}
\hline Atom & \multicolumn{1}{c}{$\mathbf{x}$} & $\mathbf{y}$ & $\mathbf{z}$ & $\boldsymbol{U}_{\boldsymbol{e q}}$ \\
\hline O2 & $7877.0(9)$ & $4303.1(14)$ & $5713.5(9)$ & $39.3(3)$ \\
N2A & $7273.8(8)$ & $6307.0(15)$ & $4774.8(10)$ & $23.5(3)$ \\
N3 & $4534.3(10)$ & $5739.9(17)$ & $3522.1(14)$ & $37.8(4)$ \\
C4 & $7615.7(10)$ & $5475.6(17)$ & $5659.9(12)$ & $24.3(3)$ \\
C5 & $7044.1(9)$ & $5863.1(17)$ & $3675.8(12)$ & $22.2(3)$ \\
C6 & $6094.0(10)$ & $6300.1(18)$ & $2759.4(12)$ & $24.8(3)$ \\
C7 & $5839.4(11)$ & $5856(2)$ & $1589.1(13)$ & $34.4(4)$ \\
C8 & $6479.3(12)$ & $6401(2)$ & $1342.6(14)$ & $37.2(4)$ \\
C9 & $7414.9(11)$ & $5993(2)$ & $2260.5(14)$ & $36.3(4)$ \\
C10 & $7676.9(10)$ & $6428(2)$ & $3427.3(13)$ & $29.7(4)$ \\
C15 & $5470.7(10)$ & $5797.0(18)$ & $3019.5(12)$ & $24.5(3)$ \\
C16 & $5296.1(9)$ & $4382.4(18)$ & $3124.8(12)$ & $25.5(3)$ \\
C17 & $4699.5(10)$ & $4400(2)$ & $3431.1(14)$ & $31.1(3)$ \\
C18 & $4998.8(10)$ & $6584.5(19)$ & $3277.6(14)$ & $30.9(4)$ \\
C19 & $4395.3(11)$ & $3196(2)$ & $3612.7(17)$ & $41.7(4)$ \\
C20 & $4703.1(12)$ & $1966(2)$ & $3509.6(18)$ & $43.3(5)$ \\
C21 & $5301.9(12)$ & $1922(2)$ & $3223.4(17)$ & $39.8(4)$ \\
C22 & $5593.6(10)$ & $3116(2)$ & $3020.8(14)$ & $32.1(4)$ \\
C23 & $4949.4(15)$ & $8096(2)$ & $3357(2)$ & $52.5(5)$ \\
O1A & $7360(3)$ & $7395(3)$ & $7587(3)$ & $34.3(4)$ \\
N1A & $7198(3)$ & $7178(4)$ & $6525(3)$ & $28.4(4)$ \\
C1A & $7639(5)$ & $6065(8)$ & $6635(3)$ & $26.9(3)$ \\
C2A & $8091(2)$ & $5519(4)$ & $7742(3)$ & 34.292 \\
C3A & $7882(2)$ & $6370(4)$ & $8294(2)$ & $34.6(4)$ \\
C11A & $8146(2)$ & $6482(3)$ & $9488(2)$ & $49.7(4)$ \\
& & & S15 & \\
& & & &
\end{tabular}




\begin{tabular}{lclrl}
\hline Atom & $\mathbf{x}$ & $\mathbf{y}$ & $\mathbf{z}$ & \multicolumn{1}{c}{$\boldsymbol{U}_{\boldsymbol{e q}}$} \\
\hline C12A & $8686(3)$ & $5213(4)$ & $10156(3)$ & $52.7(6)$ \\
C13A & $8706(3)$ & $7778(4)$ & $10040(4)$ & $52.8(7)$ \\
C14A & $7312(2)$ & $6520(5)$ & $9459(3)$ & $56.5(6)$ \\
O1B & $7368(3)$ & $7145(4)$ & $7670(3)$ & $34.3(4)$ \\
N1B & $7137(3)$ & $6931(4)$ & $6571(3)$ & $28.4(4)$ \\
C1B & $7699(6)$ & $6020(10)$ & $6684(3)$ & $26.9(3)$ \\
C2B & $8290(2)$ & $5585(4)$ & $7819(3)$ & 34.292498 \\
C3B & $8060(3)$ & $6326(5)$ & $8400(2)$ & $34.6(4)$ \\
C11B & $8319(2)$ & $6321(4)$ & $9593(3)$ & $49.7(4)$ \\
C12B & $9131(3)$ & $5413(5)$ & $10311(3)$ & $52.7(6)$ \\
C13B & $8495(4)$ & $7787(4)$ & $10061(5)$ & $52.8(7)$ \\
C14B & $7552(3)$ & $5748(6)$ & $9601(4)$ & $56.5(6)$
\end{tabular}

Table 2: Anisotropic Displacement Parameters $\left(\times 10^{4}\right)$ for KWV-II-172. The anisotropic displacement factor exponent takes the form: $-2 \pi^{2}\left[h^{2} a^{* 2} \times U_{11}+\ldots+2 h k a^{*} \times b^{*} \times U_{12}\right]$

\begin{tabular}{|c|c|c|c|c|c|c|}
\hline Atom & $U_{11}$ & $U_{22}$ & $U_{33}$ & $U_{23}$ & $U_{13}$ & $U_{12}$ \\
\hline$\overline{02}$ & $60.9(8)$ & $30.5(7)$ & $26.5(6)$ & $8.5(6)$ & $25.3(6)$ & $4.0(5)$ \\
\hline $\mathrm{N} 2 \mathrm{~A}$ & $26.2(6)$ & $26.3(7)$ & $19.0(6)$ & $-4.3(5)$ & $13.6(5)$ & $-2.7(5)$ \\
\hline N3 & $35.5(7)$ & $38.1(9)$ & $56.3(9)$ & $-1.5(7)$ & $35.9(7)$ & $-2.1(7)$ \\
\hline $\mathrm{C} 4$ & $29.2(6)$ & $25.4(8)$ & $21.9(5)$ & $-3.8(5)$ & $16.8(3)$ & $-0.9(4)$ \\
\hline C5 & 23.8(7) & $26.0(8)$ & $18.7(6)$ & $-4.6(6)$ & $13.4(6)$ & $-3.1(6)$ \\
\hline C6 & 25.1(7) & $28.7(9)$ & $20.3(7)$ & $-4.0(6)$ & $13.0(6)$ & $1.1(6)$ \\
\hline C7 & $28.0(8)$ & $51.8(11)$ & $20.0(7)$ & $-7.1(8)$ & $12.0(6)$ & $-2.9(7)$ \\
\hline C8 & $39.3(9)$ & $54.1(11)$ & $21.7(7)$ & $-7.4(9)$ & 19.6(7) & $-1.0(8)$ \\
\hline C9 & $35.1(8)$ & $53.5(12)$ & $28.2(8)$ & $-8.9(8)$ & $22.8(7)$ & $-5.5(8)$ \\
\hline C10 & $25.6(7)$ & $43.5(10)$ & $24.3(7)$ & $-7.7(7)$ & $16.8(6)$ & $-3.7(7)$ \\
\hline C15 & $23.3(7)$ & $27.2(8)$ & $25.1(7)$ & $-2.6(6)$ & $15.1(6)$ & $-1.2(6)$ \\
\hline C16 & $21.2(7)$ & $27.2(8)$ & $28.2(7)$ & $-4.2(7)$ & $14.2(6)$ & $-2.6(7)$ \\
\hline C17 & $26.8(7)$ & $31.9(9)$ & $38.6(8)$ & $-4.0(7)$ & $21.3(7)$ & $0.3(7)$ \\
\hline C18 & $29.3(8)$ & $28.2(9)$ & $39.8(9)$ & $-1.9(7)$ & $22.7(7)$ & $-1.9(7)$ \\
\hline C19 & $35.2(9)$ & $42.1(11)$ & $56.9(11)$ & $-9.5(9)$ & $31.8(9)$ & $1.2(9)$ \\
\hline $\mathrm{C} 20$ & $36.9(9)$ & $34.0(11)$ & $56.9(12)$ & $-8.4(8)$ & $25.9(9)$ & $4.1(9)$ \\
\hline $\mathrm{C} 21$ & $34.0(9)$ & $27.2(10)$ & $53.7(11)$ & $-3.1(8)$ & $22.7(8)$ & $0.0(8)$ \\
\hline $\mathrm{C} 22$ & 27.1(7) & $28.0(9)$ & $41.7(9)$ & $-3.2(7)$ & $20.3(7)$ & $-3.2(7)$ \\
\hline $\mathrm{C} 23$ & $58.5(12)$ & $34.5(11)$ & $81.4(15)$ & $0.6(9)$ & $50.1(12)$ & $-4.7(10)$ \\
\hline $01 \mathrm{~A}$ & $45.6(5)$ & $38.3(7)$ & $27.4(4)$ & $-2.5(3)$ & $26.1(2)$ & $-1.3(2)$ \\
\hline N1A & $36.4(6)$ & $30.7(8)$ & $25.8(4)$ & $-2.4(4)$ & $22.3(2)$ & $-0.3(2)$ \\
\hline C1A & $32.6(5)$ & $29.1(6)$ & $24.0(2)$ & $-4.9(3)$ & $19.23(13)$ & $-2.78(13)$ \\
\hline $\mathrm{C} 2 \mathrm{~A}$ & 40.53 & 38.14 & 25.91 & -3.05 & 20.17 & 0.45 \\
\hline $\mathrm{C} 3 \mathrm{~A}$ & $42.2(6)$ & $36.3(5)$ & $29.8(4)$ & $-5.8(2)$ & $23.5(2)$ & $-1.28(19)$ \\
\hline $\mathrm{C} 11 \mathrm{~A}$ & $68.5(7)$ & $53.2(7)$ & $33.3(4)$ & $0.2(4)$ & $32.9(2)$ & $0.2(2)$ \\
\hline $\mathrm{C} 12 \mathrm{~A}$ & 70.1(9) & $55.1(8)$ & $37.8(7)$ & $1.7(4)$ & $34.0(4)$ & $2.6(4)$ \\
\hline $\mathrm{C} 13 \mathrm{~A}$ & $73.4(11)$ & $54.5(7)$ & $37.6(10)$ & $-1.9(4)$ & $36.2(5)$ & $-2.1(3)$ \\
\hline $\mathrm{C} 14 \mathrm{~A}$ & $72.8(7)$ & $60.9(11)$ & $46.0(8)$ & $-0.0(4)$ & $40.1(3)$ & $1.6(5)$ \\
\hline 01B & $45.6(5)$ & $38.3(7)$ & $27.4(4)$ & $-2.5(3)$ & $26.1(2)$ & $-1.3(2)$ \\
\hline N1B & $36.4(6)$ & $30.7(8)$ & $25.8(4)$ & $-2.4(4)$ & $22.3(2)$ & $-0.3(2)$ \\
\hline C1B & $32.6(5)$ & $29.1(6)$ & $24.0(2)$ & $-4.9(3)$ & $19.23(13)$ & $-2.78(13)$ \\
\hline $\mathrm{C} 2 \mathrm{~B}$ & 40.53 & 38.14 & 25.91 & -3.05 & 20.17 & 0.45 \\
\hline C3B & $42.2(6)$ & $36.3(5)$ & $29.8(4)$ & $-5.8(2)$ & $23.5(2)$ & $-1.28(19)$ \\
\hline C11B & $68.5(7)$ & $53.2(7)$ & $33.3(4)$ & $0.2(4)$ & $32.9(2)$ & $0.2(2)$ \\
\hline C12B & $70.1(9)$ & $55.1(8)$ & $37.8(7)$ & $1.7(4)$ & $34.0(4)$ & $2.6(4)$ \\
\hline C13B & $73.4(11)$ & $54.5(7)$ & $37.6(10)$ & $-1.9(4)$ & $36.2(5)$ & $-2.1(3)$ \\
\hline C14B & $72.8(7)$ & $60.9(11)$ & $46.0(8)$ & $-0.0(4)$ & $40.1(3)$ & $1.6(5)$ \\
\hline
\end{tabular}


Table 3: Bond Lengths in Å for KWV-II-172.

\begin{tabular}{lll}
\hline Atom & Atom & Length/Å \\
\hline O2 & C4 & $1.228(2)$ \\
N2A & C4 & $1.327(2)$ \\
N2A & C5 & $1.4537(18)$ \\
N3 & C17 & $1.367(3)$ \\
N3 & C18 & $1.383(2)$ \\
C4 & C1A & $1.502(3)$ \\
C4 & C1B & $1.495(3)$ \\
C5 & C6 & $1.540(2)$ \\
C5 & C10 & $1.5240(19)$ \\
C6 & C7 & $1.534(2)$ \\
C6 & C15 & $1.499(2)$ \\
C7 & C8 & $1.527(2)$ \\
C8 & C9 & $1.516(3)$ \\
C9 & C10 & $1.522(2)$ \\
C15 & C16 & $1.446(2)$ \\
C15 & C18 & $1.374(2)$ \\
C16 & C17 & $1.418(2)$ \\
C16 & C22 & $1.398(3)$ \\
C17 & C19 & $1.394(3)$ \\
C18 & C23 & $1.486(3)$ \\
C19 & C20 & $1.375(3)$
\end{tabular}

\begin{tabular}{lll}
\hline Atom & Atom & Length/Å \\
\hline C20 & C21 & $1.400(3)$ \\
C21 & C22 & $1.387(3)$ \\
01A & N1A & $1.400(3)$ \\
O1A & C3A & $1.358(3)$ \\
N1A & C1A & $1.316(3)$ \\
C1A & C2A & $1.415(3)$ \\
C2A & C3A & $1.356(3)$ \\
C3A & C11A & $1.503(3)$ \\
C11A & C12A & $1.535(2)$ \\
C11A & C13A & $1.538(2)$ \\
C11A & C14A & $1.535(2)$ \\
O1B & N1B & $1.402(3)$ \\
O1B & C3B & $1.359(3)$ \\
N1B & C1B & $1.312(3)$ \\
C1B & C2B & $1.415(3)$ \\
C2B & C3B & $1.354(3)$ \\
C3B & C11B & $1.500(3)$ \\
C11B & C12B & $1.531(3)$ \\
C11B & C13B & $1.535(3)$ \\
C11B & C14B & $1.543(3)$
\end{tabular}

Table 4: Bond Angles in ${ }^{\circ}$ for KWV-II-172.

\begin{tabular}{llll}
\hline Atom & Atom & Atom & \multicolumn{1}{c}{ Angle $^{\circ}$} \\
\hline C5 & N2A & C4 & $123.19(14)$ \\
C18 & N3 & C17 & $109.58(14)$ \\
N2A & C4 & O2 & $125.60(13)$ \\
C1A & C4 & O2 & $120.2(2)$ \\
C1A & C4 & N2A & $114.2(2)$ \\
C1B & C4 & O2 & $117.3(3)$ \\
C1B & C4 & N2A & $117.1(3)$ \\
C1B & C4 & C1A & $3.9(4)$ \\
C6 & C5 & N2A & $108.95(12)$ \\
C10 & C5 & N2A & $111.82(12)$ \\
C10 & C5 & C6 & $111.25(12)$ \\
C7 & C6 & C5 & $109.93(13)$ \\
C15 & C6 & C5 & $111.69(12)$ \\
C15 & C6 & C7 & $113.24(13)$ \\
C8 & C7 & C6 & $111.99(14)$ \\
C9 & C8 & C7 & $111.50(14)$ \\
C10 & C9 & C8 & $111.61(14)$ \\
C9 & C10 & C5 & $111.41(13)$ \\
C16 & C15 & C6 & $126.47(14)$ \\
C18 & C15 & C6 & $126.82(16)$ \\
C18 & C15 & C16 & $106.59(14)$ \\
C17 & C16 & C15 & $106.71(14)$ \\
C22 & C16 & C15 & $134.73(14)$ \\
C22 & C16 & C17 & $118.53(15)$ \\
C16 & C17 & N3 & $107.68(15)$ \\
C19 & C17 & N3 & $130.38(16)$ \\
& & &
\end{tabular}

\begin{tabular}{llll}
\hline Atom & Atom & Atom & Angle $^{\circ}$ \\
\hline C19 & C17 & C16 & $121.92(17)$ \\
C15 & C18 & N3 & $109.43(16)$ \\
C23 & C18 & N3 & $119.67(16)$ \\
C23 & C18 & C15 & $130.87(17)$ \\
C20 & C19 & C17 & $118.20(16)$ \\
C21 & C20 & C19 & $120.99(18)$ \\
C22 & C21 & C20 & $121.00(18)$ \\
C21 & C22 & C16 & $119.34(15)$ \\
C3A & O1A & N1A & $108.8(2)$ \\
C1A & N1A & O1A & $105.5(2)$ \\
N1A & C1A & C4 & $121.7(3)$ \\
C2A & C1A & C4 & $126.5(3)$ \\
C2A & C1A & N1A & $111.7(2)$ \\
C3A & C2A & C1A & $104.5(2)$ \\
C2A & C3A & O1A & $109.4(2)$ \\
C11A & C3A & O1A & $115.4(2)$ \\
C11A & C3A & C2A & $135.0(3)$ \\
C12A & C11A & C3A & $108.9(2)$ \\
C13A & C11A & C3A & $109.3(3)$ \\
C13A & C11A & C12A & $109.5(3)$ \\
C14A & C11A & C3A & $108.5(2)$ \\
C14A & C11A & C12A & $109.0(3)$ \\
C14A & C11A & C13A & $111.7(3)$ \\
C3B & O1B & N1B & $109.1(3)$ \\
C1B & N1B & O1B & $105.0(2)$ \\
N1B & C1B & C4 & $120.0(3)$ \\
& & &
\end{tabular}




\begin{tabular}{llll}
\hline Atom & Atom & Atom & Angle $^{\circ}$ \\
\hline C2B & C1B & C4 & $127.5(3)$ \\
C2B & C1B & N1B & $112.3(2)$ \\
C3B & C2B & C1B & $104.3(2)$ \\
C2B & C3B & 01B & $109.3(2)$ \\
C11B & C3B & 01B & $115.3(3)$ \\
C11B & C3B & C2B & $134.9(3)$
\end{tabular}

\begin{tabular}{lllr}
\hline Atom & Atom & Atom & Angle $^{\circ}$ \\
\hline C12B & C11B & C3B & $108.5(3)$ \\
C13B & C11B & C3B & $110.4(3)$ \\
C13B & C11B & C12B & $111.3(3)$ \\
C14B & C11B & C3B & $109.0(3)$ \\
C14B & C11B & C12B & $110.2(3)$ \\
C14B & C11B & C13B & $107.3(3)$
\end{tabular}

Table 5: Torsion Angles in ${ }^{\circ}$ for KWV-II-172.

\begin{tabular}{|c|c|c|c|c|}
\hline Atom & Atom & Atom & Atom & Angle $/^{\circ}$ \\
\hline 02 & $\mathrm{C} 4$ & $\mathrm{~N} 2 \mathrm{~A}$ & C5 & $9.3(2)$ \\
\hline 02 & C4 & $\mathrm{C} 1 \mathrm{~A}$ & N1A & $-164.9(6)$ \\
\hline 02 & C4 & $\mathrm{C} 1 \mathrm{~A}$ & $\mathrm{C} 2 \mathrm{~A}$ & $13.2(2)$ \\
\hline 02 & $\mathrm{C} 4$ & C1B & N1B & $-150.5(7)$ \\
\hline 02 & $\mathrm{C} 4$ & $\mathrm{C} 1 \mathrm{~B}$ & $\mathrm{C} 2 \mathrm{~B}$ & $23.5(3)$ \\
\hline N2A & $\mathrm{C} 4$ & C1A & N1A & $13.1(3)$ \\
\hline N2A & C4 & C1A & $\mathrm{C} 2 \mathrm{~A}$ & $-168.8(5)$ \\
\hline $\mathrm{N} 2 \mathrm{~A}$ & C4 & $\mathrm{C} 1 \mathrm{~B}$ & N1B & $30.2(3)$ \\
\hline $\mathrm{N} 2 \mathrm{~A}$ & C4 & $\mathrm{C} 1 \mathrm{~B}$ & $\mathrm{C} 2 \mathrm{~B}$ & $-155.8(6)$ \\
\hline $\mathrm{N} 2 \mathrm{~A}$ & $\mathrm{C} 5$ & C6 & C7 & $179.39(13)$ \\
\hline $\mathrm{N} 2 \mathrm{~A}$ & C5 & C6 & C15 & $-54.02(14)$ \\
\hline N2A & $\mathrm{C} 5$ & C10 & C9 & $-178.27(15)$ \\
\hline N3 & C17 & C16 & C15 & $-0.85(15)$ \\
\hline N3 & C17 & C16 & $\mathrm{C} 22$ & $177.63(13)$ \\
\hline N3 & C17 & C19 & $\mathrm{C} 20$ & $-177.0(2)$ \\
\hline N3 & C18 & C15 & C6 & $-176.85(12)$ \\
\hline N3 & C18 & C15 & C16 & $-0.75(15)$ \\
\hline C4 & $\mathrm{C} 1 \mathrm{~A}$ & N1A & 01A & $178.7(7)$ \\
\hline C4 & C1A & $\mathrm{C} 2 \mathrm{~A}$ & C3A & $-177.4(8)$ \\
\hline C4 & $\mathrm{C} 1 \mathrm{~B}$ & N1B & 01B & $176.3(8)$ \\
\hline C4 & C1B & $\mathrm{C} 2 \mathrm{~B}$ & C3B & $-175.8(10)$ \\
\hline C5 & C6 & $\mathrm{C} 7$ & C8 & $-55.09(16)$ \\
\hline C5 & C6 & C15 & C16 & $-61.51(15)$ \\
\hline C5 & C6 & C15 & C18 & $113.84(15)$ \\
\hline C5 & C10 & C9 & C8 & $55.33(17)$ \\
\hline C6 & C7 & C8 & C9 & $54.90(18)$ \\
\hline C6 & C15 & C16 & C17 & $177.10(16)$ \\
\hline C6 & C15 & C16 & $\mathrm{C} 22$ & $-1.0(2)$ \\
\hline C6 & C15 & C18 & $\mathrm{C} 23$ & $1.1(2)$ \\
\hline C7 & $\mathrm{C} 8$ & $\mathrm{C} 9$ & $\mathrm{C} 10$ & $-54.38(19)$ \\
\hline C15 & C16 & C17 & C19 & $-179.46(13)$ \\
\hline C15 & C16 & $\mathrm{C} 22$ & $\mathrm{C} 21$ & $177.69(19)$ \\
\hline C16 & C17 & C19 & $\mathrm{C} 20$ & $1.3(2)$ \\
\hline C16 & $\mathrm{C} 22$ & $\mathrm{C} 21$ & $\mathrm{C} 20$ & $1.2(2)$ \\
\hline C17 & C19 & $\mathrm{C} 20$ & $\mathrm{C} 21$ & $-0.4(2)$ \\
\hline C19 & $\mathrm{C} 20$ & $\mathrm{C} 21$ & $\mathrm{C} 22$ & $-0.9(2)$ \\
\hline $01 \mathrm{~A}$ & N1A & $\mathrm{C} 1 \mathrm{~A}$ & $\mathrm{C} 2 \mathrm{~A}$ & $0.3(4)$ \\
\hline $01 \mathrm{~A}$ & $\mathrm{C} 3 \mathrm{~A}$ & $\mathrm{C} 2 \mathrm{~A}$ & C1A & $-1.8(6)$ \\
\hline $01 \mathrm{~A}$ & C3A & C11A & $\mathrm{C} 12 \mathrm{~A}$ & $176.8(4)$ \\
\hline $01 \mathrm{~A}$ & C3A & C11A & C13A & $-63.7(4)$ \\
\hline $01 \mathrm{~A}$ & C3A & $\mathrm{C} 11 \mathrm{~A}$ & $\mathrm{C} 14 \mathrm{~A}$ & $58.3(4)$ \\
\hline N1A & $\mathrm{C} 1 \mathrm{~A}$ & $\mathrm{C} 2 \mathrm{~A}$ & C3A & $0.9(8)$ \\
\hline C1A & $\mathrm{C} 2 \mathrm{~A}$ & C3A & C11A & $-176.3(5)$ \\
\hline $\mathrm{C} 2 \mathrm{~A}$ & C3A & $\mathrm{C} 11 \mathrm{~A}$ & $\mathrm{C} 12 \mathrm{~A}$ & $-8.9(5)$ \\
\hline $\mathrm{C} 2 \mathrm{~A}$ & C3A & $\mathrm{C} 11 \mathrm{~A}$ & $\mathrm{C} 13 \mathrm{~A}$ & $110.6(5)$ \\
\hline
\end{tabular}




\begin{tabular}{llllr}
\hline Atom & Atom & Atom & Atom & Angle $^{\circ}$ \\
\hline C2A & C3A & C11A & C14A & $-127.4(5)$ \\
O1B & N1B & C1B & C2B & $1.4(5)$ \\
O1B & C3B & C2B & C1B & $0.8(7)$ \\
O1B & C3B & C11B & C12B & $-177.1(4)$ \\
01B & C3B & C11B & C13B & $-54.9(5)$ \\
O1B & C3B & C11B & C14B & $62.8(5)$ \\
N1B & C1B & C2B & C3B & $-1.5(10)$ \\
C1B & C2B & C3B & C11B & $172.3(6)$ \\
C2B & C3B & C11B & C12B & $11.8(7)$ \\
C2B & C3B & C11B & C13B & $134.1(6)$ \\
C2B & C3B & C11B & C14B & $-108.3(6)$
\end{tabular}

Table 6: Hydrogen Fractional Atomic Coordinates $\left(\times 10^{4}\right)$ and Equivalent Isotropic Displacement Parameters $\left(\AA^{2} \times 10^{3}\right)$ for KWV-II-172. $U_{e q}$ is defined as $1 / 3$ of the trace of the orthogonalised $U_{i j}$.

\begin{tabular}{|c|c|c|c|c|}
\hline Atom & $\mathbf{x}$ & $\mathbf{y}$ & $\mathbf{z}$ & $U_{e q}$ \\
\hline $\mathrm{H} 5$ & $7075(13)$ & $4760(20)$ & $3716(17)$ & $40(5)$ \\
\hline H6 & $6080(14)$ & $7420(30)$ & $2759(18)$ & $45(5)$ \\
\hline $\mathrm{H7a}$ & $5164(6)$ & $6200(30)$ & $951(14)$ & $45(5)$ \\
\hline $\mathrm{H} 7 \mathrm{~b}$ & $5856(15)$ & $4730(3)$ & $1569(19)$ & $50(6)$ \\
\hline H8a & $6298(14)$ & $6000(20)$ & $524(10)$ & $54(6)$ \\
\hline $\mathrm{H} 8 \mathrm{~b}$ & $6407(16)$ & $7522(4)$ & $1300(20)$ & $53(7)$ \\
\hline H9a & $7842(12)$ & $6460(20)$ & $2055(18)$ & $50(6)$ \\
\hline $\mathrm{H} 9 \mathrm{~b}$ & $7461(16)$ & $4873(4)$ & $2230(20)$ & $63(7)$ \\
\hline H10a & $8329(8)$ & $6020(30)$ & $4082(15)$ & $55(6)$ \\
\hline $\mathrm{H} 10 \mathrm{~b}$ & $7652(15)$ & $7550(4)$ & $3475(19)$ & $47(6)$ \\
\hline H19 & 3965(18) & $3300(30)$ & $3870(20)$ & $75(8)$ \\
\hline $\mathrm{H} 20$ & $4472(19)$ & $1050(30)$ & $3670(20)$ & $75(9)$ \\
\hline H21 & $5546(16)$ & $910(30)$ & $3140(20)$ & $58(7)$ \\
\hline H22 & $6074(16)$ & $3090(30)$ & $2820(20)$ & $58(6)$ \\
\hline $\mathrm{H} 23 \mathrm{a}$ & 5359.4(15) & $8599(2)$ & $3134(2)$ & $170(12)$ \\
\hline $\mathrm{H} 23 \mathrm{~b}$ & $4262.8(16)$ & $8434(2)$ & $2767(2)$ & $170(12)$ \\
\hline H23c & 5189.3(15) & $8377(2)$ & $4230(2)$ & $170(12)$ \\
\hline $\mathrm{H} 2 \mathrm{Aa}$ & $8460(20)$ & $4640(20)$ & $7990(30)$ & 41.151 \\
\hline $\mathrm{H} 12 \mathrm{a}$ & $8256(3)$ & $4303(4)$ & $9847(3)$ & 79.1(9) \\
\hline $\mathrm{H} 12 \mathrm{~b}$ & $9213(3)$ & $5058(4)$ & $10035(3)$ & $79.1(9)$ \\
\hline $\mathrm{H} 12 \mathrm{c}$ & $8978(3)$ & $5366(4)$ & 11064(3) & 79.1(9) \\
\hline H13a & $9340(3)$ & $7650(4)$ & $10155(4)$ & $79.2(10)$ \\
\hline $\mathrm{H} 13 \mathrm{~b}$ & $8357(3)$ & $8672(4)$ & $9490(4)$ & $79.2(10)$ \\
\hline H13c & $8823(3)$ & 7944(4) & 10872(4) & $79.2(10)$ \\
\hline $\mathrm{H} 14 \mathrm{a}$ & $6972(2)$ & $7508(5)$ & $9101(3)$ & $84.8(9)$ \\
\hline $\mathrm{H} 14 \mathrm{~b}$ & $6874(2)$ & $5672(5)$ & $8927(3)$ & $84.8(9)$ \\
\hline $\mathrm{H} 14 \mathrm{c}$ & $7489(2)$ & $6408(5)$ & $10327(3)$ & $84.8(9)$ \\
\hline H2B & $8778(3)$ & $4873(5)$ & $8129(3)$ & 41.151 \\
\hline H12d & $8960(3)$ & 4321(6) & 9998(3) & 79.1(9) \\
\hline $\mathrm{H} 12 \mathrm{e}$ & $9668(3)$ & 5793(5) & 10241(3) & 79.1(9) \\
\hline $\mathrm{H} 12 \mathrm{f}$ & $9363(3)$ & $5456(5)$ & $11225(4)$ & $79.1(9)$ \\
\hline H13d & $9039(4)$ & $8256(4)$ & $10043(5)$ & $79.2(10)$ \\
\hline $\mathrm{H} 13 \mathrm{e}$ & $7881(4)$ & $8422(5)$ & $9524(5)$ & $79.2(10)$ \\
\hline $\mathrm{H} 13 \mathrm{f}$ & $8695(4)$ & $7763(4)$ & $10962(5)$ & $79.2(10)$ \\
\hline H14d & 6938(3) & $6355(6)$ & 8991(4) & $84.8(9)$ \\
\hline $\mathrm{H} 14 \mathrm{e}$ & $7442(3)$ & $4636(6)$ & 9335(4) & $84.8(9)$ \\
\hline $\mathrm{H} 14 \mathrm{f}$ & $7716(3)$ & $5835(6)$ & $10482(4)$ & $84.8(9)$ \\
\hline $\mathrm{H} 2 \mathrm{~A}$ & $7134(15)$ & $7260(30)$ & $4860(20)$ & $47(6)$ \\
\hline H3 & $4120(20)$ & $6090(30)$ & $3710(20)$ & $73(8)$ \\
\hline
\end{tabular}


Table 7: Atomic Occupancies for all atoms that are not fully occupied in KWV-II-172.

\begin{tabular}{lr}
\hline Atom & Occupancy \\
\hline O1A & 0.542820 \\
N1A & 0.542820 \\
C1A & 0.542820 \\
C2A & 0.542820 \\
H2Aa & 0.542820 \\
C3A & 0.542820 \\
C11A & 0.542820 \\
C12A & 0.542820 \\
H12a & 0.542820 \\
H12b & 0.542820 \\
H12c & 0.542820 \\
C13A & 0.542820 \\
H13a & 0.542820 \\
H13b & 0.542820 \\
H13c & 0.542820 \\
C14A & 0.542820 \\
H14a & 0.542820 \\
H14b & 0.542820 \\
H14c & 0.542820 \\
O1B & 0.457180 \\
N1B & 0.457180 \\
C1B & 0.457180 \\
C2B & 0.457180 \\
H2B & 0.457180 \\
C3B & 0.457180 \\
C11B & 0.457180 \\
C12B & 0.457180 \\
H12d & 0.457180 \\
H12e & 0.457180 \\
H12f & 0.457180
\end{tabular}

\section{References}

Spek, A. L. Single-Crystal Structure Validation with the Program PLATON. J. Appl. Cryst. 2003, 36, 7-13.

APEX2 suite for crystallographic software, Bruker axs, Madison, WI.

Dolomanov, O. V.; Bourhis, L. J.; Gildea, R. J.; Howard, J. A. K.; Puschmann, H. Olex2: A Complete Structure Solution, Refinement and Analysis Program. J. Appl. Cryst., 2009, 42, 339-341.

SADABS-2014/5, Bruker axs, Madison, WI (2014).

SAINT-8.34A-2013 - Software for the Integration of CCD Detector System Bruker Analytical X-ray Systems, Bruker axs, Madison, WI (2013).

Sheldrick, G. M. A Short History of ShelX. Acta Cryst., 2008, A64, 339-341.

Sheldrick, G. M. ShelXT-Integrated Space-Group and Crystal-Structure Determination. Acta Cryst., 2015, A71, 3-8. 